\title{
A fast L1 adaptive constrained back-stepping controller using barrier Lyapunov function for anuncertain submersible vehicle
}

Hossein Ahmadian

Amirkabir University of Technology

Mehdi Arefi

Shiraz University

Alireza Khayatian

Shiraz University

Allahyar Montazeri ( $\square$ a.montazeri@lancaster.ac.uk)

Lancaster University

\section{Research Article}

Keywords: adaptive, controller, proposed, results, tracking

Posted Date: September 7th, 2021

DOI: https://doi.org/10.21203/rs.3.rs-866911/v1

License: (c) (i) This work is licensed under a Creative Commons Attribution 4.0 International License.

Read Full License 


\title{
A fast $\mathscr{L}_{1}$ adaptive constrained back-stepping controller using barrier Lyapunov function for an uncertain submersible vehicle
}

\author{
Hossein Ahmadian ${ }^{1}$, Mohammad Mahdi Arefi ${ }^{2}$, Alireza Khayatian ${ }^{2}$, and Allahyar \\ Montazeri, ${ }^{3, *}$
}

\author{
${ }^{1}$ Amirkabir University of Technology, Department of Electrical Engineering, Tehran, Iran. \\ ${ }^{2}$ Shiraz University, Department of Power and Control Engineering, Shiraz, Iran. \\ ${ }^{3}$ Lancaster University, Engineering Department, Lancaster, United Kingdom. \\ *a.montazeri@lancaster.ac.uk
}

\begin{abstract}
In this paper, a new $\mathscr{L}_{1}$ adaptive back-stepping controller based on the barrier Lyapunov function (BLF) is proposed to respect the position and velocity constraints usually imposed in designing Euler-Lagrange systems. The purpose of this investigation is to improve different aspects of conventional $\mathscr{L}_{1}$ adaptive control. More specifically, the modified controller has a lower complexity by removing the low-pass filter from the design procedure. The performance of the controller is also enhanced by having a faster convergence speed and increased robustness against nonlinear uncertainties and disturbances arising in practical applications. The proposed scheme is evaluated on two different Euler-Lagrange systems, i.e. a 6-DOF remotely operated vehicle (ROV) and a single-link manipulator. The results for the new back-stepping design are assessed in both scenarios in terms of settling time, percentage of overshoot, and trajectory tracking error. The results confirm that both tracking and state estimation errors for position and velocity outputs outperform the standard $\mathscr{L}_{1}$ adaptive control technique. The results also demonstrate the high performance of the proposed approach in removing the matched nonlinear time-varying disturbances and dynamic uncertainties and a good trajectory tracking despite the uncertainty on the input gain of the system.
\end{abstract}

\section{Introduction}

The use of robotics and autonomous systems in hazardous environments, which are not easily accessible to human have been the subject of study in various fields $s^{1,2,3}$. One of the hazardous environments that human being has not been able to discover effectively is the deep see and ocean surfaces. The underwater robots have been proven a useful technology to explore and investigate such inaccessible environments ${ }^{4,5}$. Moreover, in nuclear applications underwater robots have shown a great potential for inspection and monitoring of the environments. Here, we look at a specific category of underwater robots, called submersible remotely operated vehicles (ROVs). The global offshore ROV industry is predicted to be worth up to $\$ 3.5 \mathrm{Bn}$ with modern applications including civil and inshore inspections (CIIs) and decommissioning of oil and gas pipelines, nuclear storage facilities and accident sites, liquid storage tanks and tunnels. A number of small, relatively cheap, commercially available ROVs exist, which may be useful for decommissioning and inspection activities within the inaccessible submerged sites found in the nuclear industry. For example, in decommissioning applications, ROVs are often utilised to deploy customised sensors and tooling to support activities such as surveying of nuclear ponds, sampling of sludge in ponds, and remote handling tasks 5 .

Autonomous operation of this sort of robots raises various control problems. The problems are originated from the intrinsic time-varying and high level of nonlinearities, resulting from the hydrodynamic effects and the high level of external disturbance, caused by the sea currents and the drag effects acting on the vehicle ${ }^{6,7}$. To overcome these problems, various control methods have been proposed in the literature such as adaptive RISE ${ }^{8}$, feedback linearization controller ${ }^{9}$, output feedback control ${ }^{10}, \mathscr{L}_{1}$ adaptive controller ${ }^{11,12}$, and adaptive fuzzy control ${ }^{13}$, Lyapunov-based model predictive control ${ }^{14}$, robust $\mathscr{H}_{\infty}$ control $^{15}$, fuzzy backstepping sliding mode control ${ }^{16}$. A more detailed review of the proposed controllers for AUVs can be found $\mathrm{in}^{17}$. In all of the above-mentioned articles, the requirement for adaptation and robustness is of key importance. This is due to the fact that identifying the robot's parameters are usually difficult and they are subject to rapid changes due to the varying conditions of the environment that they operate. Adaptive controllers are well known to work efficiently with respect to the changes in the parameters and uncertainties, occurring in the real world $10,18,19,20$.

The main incentive of using $\mathscr{L}_{1}$ adaptive control is its ability to cope with unmatched and matched uncertainties as well as the possibility of estimating uncertainties and determining the control effort to minimize the influence of these uncertainties on 
the system closed-loop response. The low-pass filters, introduced in this method, even in the presence of fast adaptation and large amplitude reference inputs, guarantees that the closed-loop response of $\mathscr{L}_{1}$ adaptive control output remains in the lowfrequency range ${ }^{21,22}$. The primary results on $\mathscr{L}_{1}$ adaptive control system design are reported in the seminal work in ${ }^{23}$. In this work, the problem formulation of $\mathscr{L}_{1}$ adaptive control, initial theories, and the stability analysis results for the controller have been derived. Practical implementation of $\mathscr{L}_{1}$ adaptive controller on a flexible launch vehicle is reported a few years later in ${ }^{24}$. A more detailed explanation of the $\mathscr{L}_{1}$ adaptive control theory as well as the ideas about adaptive flight control applications is reported $i^{25}$ by the instigators of the $\mathscr{L}_{1}$ adaptive control. Furthermore, the $\mathscr{L}_{1}$ adaptive controller has been applied to minimum-phase systems based on output feedback control system theories $i^{26}$. Also, a multi-criteria design scheme, based on greedy search method has been proposed $\mathrm{in}^{27}$ to design the parameters of the low-pass filter, existing in the process of the controller design.

Application of $\mathscr{L}_{1}$ adaptive controller along with other forms of controllers to improve the dynamic performance of the underwater vehicles has been reported in a numerous paper. For example, the use of feedback linearization for direct control of underwater vehicle-manipulator systems (UVMS) is suggested in ${ }^{9}$. In this paper, the non-linear dynamic of the system is replaced with a linear system and the control rule is chosen to force the tracking error to zero. The challenge in using the feedback linearization controller is the exact knowledge of the system under control. The UVMS is a strongly non-linear system and has unmodeled dynamics, therefore, the feedback linearization controller is not an appropriate choice. In ${ }^{28}$ an adaptive controller for trajectory tracking problem in UVMS is proposed without using any straight velocity feedback. Here, the velocity is estimated by designing an observer to be used in designing the control law. Therefore, by designing an observer and a controller the exponential convergence of the trajectory tracking problem as well as convergence of the estimation error to zero is guaranteed. ${ }^{10}$ has studied the kinematic and dynamic of UVMSs. In this work, the performance of four different controllers, i.e. adaptive control, feedback linearization, output feedback control, and sliding mode control, have been compared by considering uncertainties and ocean wave current. The basic concepts in modelling of kinematics and dynamics of autonomous underwater vehicles (AUV) are studied by ${ }^{29}$. Therefore, the isolated movements of the AUV are studied by controlling its lateral and collective speeds and positions. Here, the performance of the whole system, is evaluated using both experimental and simulation work. $\operatorname{In}^{30}$ has proposed the software and hardware architecture for guidance and control system of a specific type of AUV, , i.e. AUV-150. In [26], the $\mathscr{L}_{1}$ adaptive controller is utilised to control the pitch angle and depth of a 6 degree of freedom ROV. This controller is applied to the real AC-ROV to compensate the effect of external disturbances and changes in the system parameters. A study, carried out in ${ }^{31}$, compares the performance of a common adaptive control method with an $\mathscr{L}_{1}$ adaptive control by applying both controllers to a Rohrs and two carts as the benchmark problems.

The $\mathscr{L}_{1}$ adaptive controller has two fundamental architectures (i) state feedback setting, and (ii) output feedback setting ${ }^{4}$. An $\mathscr{L}_{1}$ adaptive output feedback control architecture for an underactuated MIMO system has been developed in ${ }^{32}$. The main issues to design such a system are unknown input gain, non-square structure, and the existing uncertainties. In this work, it is shown that, the suggested control scheme, guarantees the boundedness of the error signal. The trajectory tracking problem in the presence of modelling uncertainties as well as the wind and earth effects as the external disturbances is studied in ${ }^{33}$. Since no uncertainty in the kinematics is considered in this work, the $\mathscr{L}_{1}$ adaptive backstepping control method has shown the desired performance. A decentralized control design for unmodeled nonlinear dynamics of a $1.5 \mathrm{MW}$ wind turbine is proposed $\mathrm{in}^{34}$. The scheme has implemented a fuzzy logic system (for estimation of nonlinearities and compensating the effect of uncertainties) along with the $\mathscr{L}_{1}$ adaptive controller. $\mathrm{In}^{35}$, the tracking control problem of a flying manipulator, coupled with a quadrotor, has been addressed. In this paper, the analytical model of the coupled system is developed and the uncertainties between the robotic arm and the aerial platform are formulated as a time-varying nonlinear uncertainty. Then, the $\mathscr{L}_{1}$ adaptive controller is proposed to control the main loop and compensate the effect of uncertainties and ensure the desired trajectory tracking performance. Moreover, ${ }^{36}$ has proposed a new controller design for a MIMO two-link robot manipulator with disturbances and non-linearity by combining fuzzy PDC and $\mathscr{L}_{1}$ adaptive control schemes. For unmanned underwater vehicle applications, ${ }^{37}$ has developed a new integral sliding mode controller for a system with unknown disturbances and nonlinear uncertainties based on an MIMO extended state observer. The concept of barrier Lyapunov function (BLF) has been pursued particularly for the collision avoidance problem in robotics applications. However, ${ }^{38}$ proposed a robust adaptive controller using the velocity constraints for a ROV. In this work, the model parameters are determined in real-time and a BLF is used in accordance with the Lyapunov theory.

In this study, the problem of $\mathscr{L}_{1}$ adaptive back-stepping controller using BLF for a class of dynamic systems with uncertain nonlinear functions, inaccessible states, uncertainty on input gain and unknown disturbance is investigated. Here, we assumed an unknown disturbance in all of our analyses. To respect the practical limitations, some hard constraints on the position and velocity errors are considered. Having these constraints and the definition of Lyapunov function as a BLF, a novel controller with a new adaptation law is proposed and the conditions for stability of the closed-loop system is provided. Such a design removes the necessity of designing the low-pass filter in the $\mathscr{L}_{1}$ adaptive method, while other features of the controller are 
maintained. Furthermore, the proposed method guarantees that the tracking error of the controller stays inside predefined hard-bound constraints, despite the unknown system parameters and dynamic uncertainties of the system. The performance of the proposed controller is evaluated numerically on a 6-DOF ROV with a single-link manipulator in two case studies. The results, confirm that the proposed method can successfully eliminate the effect of time-varying disturbances and uncertainties, and satisfies the tracking performance bounds despite the nonlinearities.

This paper is organized as follows. In section 2, the model and characteristics of the submersible ROV are introduced, and then the nonlinear dynamic and kinematic equations of the system are derived. The problem formulation, theoretical background of the $\mathscr{L}_{1}$ adaptive controller, definition of BLF as well as design and stability proof of the proposed $\mathscr{L}_{1}$ adaptive backstepping controller are presented in section 3 . In section 4 , the numerical simulations and analysis of the case studies are discussed. Finally, the paper is wrapped up in section 5.

\section{System descriptions}

During the past 40 years, the increasing demands for oceanic drawing, sea discovery as well as oil and gas exploitation has led to the development of an underwater vehicle that can be controlled from long distance. Remotely operated underwater vehicle are unmanned vehicles, operated by a person working on a vessel or on the land. Submersible ROVs are usually tethered by using a cable that transmits the electrical power. ROVs are used in a variety of fields such as scientific research, underwater archaeology, oil and gas drilling support, reconnaissance, homeland security, military applications, and telecommunications. The schematic model of a typical ROV with the assigned coordinate systems is illustrated in Figure 1.

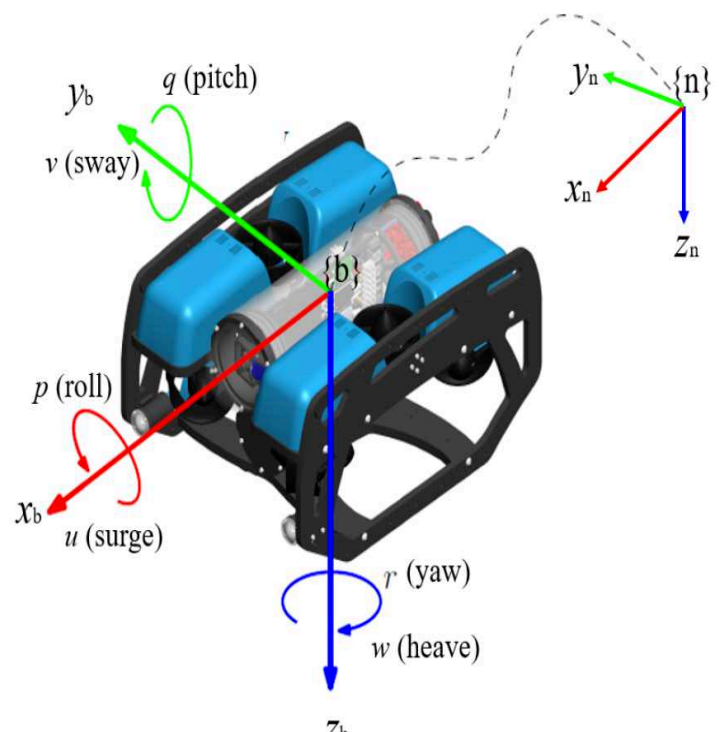

Figure 1. The schematic of a typical ROV with coordinate system ${ }^{12}$.

\section{Nonlinear Dynamic and Kinematic Model}

The dynamic model of the underwater robot is represented in the following general form ${ }^{39}$

$$
\begin{aligned}
& \mathbf{M} \dot{\mathbf{v}}+\mathbf{C}(\mathbf{v}) \mathbf{v}+\mathbf{D v}+\mathbf{g}(\eta)=\mathbf{u} \\
& \dot{\eta}=\mathbf{J}(\eta) \mathbf{v}
\end{aligned}
$$

In (1), $\mathbf{M}, \mathbf{C}$ and $\mathbf{D}$ are the inertia matrix, the centrifugal matrix (Coriolis), the damping matrix, and the vector of gravity term, respectively. Besides, $J(\eta) \in \mathbb{R}^{6 \times 6}$ is the transformation matrix, mapping the body-fixed frame to the earth-fixed frame, and $\mathbf{u}$ is the control input vector. The vectors $\mathbf{v}$ and $\eta$ are velocity and position of the underwater vehicle, in two different coordinate systems, respectively. The elements of the velocity vector $\mathbf{v}$ in the body frame are $u, v, w, p, q$ and $r$ as shown in Figure 1. Moreover, the elements of the position vector $\eta$ in the world frame are $x, y, z, \phi, \theta$ and $\psi$. Equation (1), represents the dynamics of the 6-DOF system with 3 translational and 3 rotational dynamics. Therefore, the dynamic equations of the system can be written as

$$
\begin{aligned}
& \mathbf{M} \dot{\mathbf{v}}+\mathbf{C}(\mathbf{v}) \mathbf{v}+\left(\mathbf{D}_{\mathbf{L}}+\mathbf{D}_{\mathbf{n L}}(\mathbf{v})\right) \mathbf{v}+\mathbf{g}(\eta)=\mathbf{u} \\
& \dot{\eta}=\mathbf{J}(\eta) \mathbf{v}
\end{aligned}
$$


By defining $\mathbf{X}=\left[\mathbf{x}_{\mathbf{1}}=\eta, \mathbf{x}_{\mathbf{2}}=\mathbf{v}\right]^{\mathbf{T}}$ as the system state vector, the state space model of the system can be derived as

$$
\begin{aligned}
& \dot{\mathbf{x}}_{\mathbf{1}}=\mathbf{J}\left(\mathbf{x}_{\mathbf{1}}\right) \mathbf{x}_{\mathbf{2}} \\
& \dot{\mathbf{x}}_{\mathbf{2}}=-\mathbf{M}^{-\mathbf{1}} \mathbf{D}_{\mathbf{L}} \mathbf{x}_{\mathbf{2}}-\mathbf{M}^{-\mathbf{1}}\left(\mathbf{C}\left(\mathbf{x}_{\mathbf{2}}\right)+\mathbf{D}_{\mathbf{n L}}\left(\mathbf{x}_{\mathbf{2}}\right)\right) \mathbf{x}_{\mathbf{2}}-\mathbf{M}^{-\mathbf{1}} \mathbf{g}\left(\mathbf{x}_{\mathbf{1}}\right)+\mathbf{M}^{-\mathbf{1}} \mathbf{u},
\end{aligned}
$$

where, $\mathbf{x}_{\mathbf{1}}=[x, y, z, \phi, \theta, \psi]^{T}$ and $\mathbf{x}_{\mathbf{2}}=[u, v, w, p, q, r]^{T}$.

Remark 1. Unlike the background work carried out in ${ }^{12}$, the underwater vehicle doesn't have a slow dynamic. Also, the Coriolis term $\mathbf{C}(\mathbf{v})$ is non-zero and the damping matrix is considered as a combination of linear and non-linear damping, i.e. $\mathbf{D}(\mathbf{v})=\mathbf{D}_{\mathbf{L}}+\mathbf{D}_{\mathbf{n} \mathbf{L}}(\mathbf{v})$. It is assumed that the damping matrix is a real positive definite asymmetric matrix. Since $\mathbf{C}(\mathbf{v})$ is non-zero in (2), the ROV is assumed to have a high-velocity and non-adjustable motion.

\section{Preliminaries}

\section{Basic structure}

Consider the MIMO system with the dynamic equations represented in (4)

$$
\begin{aligned}
& \dot{\mathbf{x}}(t)=\mathbf{A x}(t)+\mathbf{B u}(t)+\mathbf{f}(t, \mathbf{x}(t)) \\
& \mathbf{y}(t)=\mathbf{C x}(t) .
\end{aligned}
$$

In (4), $\mathbf{x}(t) \in \mathbb{R}^{2 n}$ is the state vector of the system with $\mathbf{x}(0)=\mathbf{x}_{0}, \mathbf{u}(t) \in \mathbb{R}^{m}$ is the control input vector $(m \leq 2 n), \mathbf{A} \in$ $\mathbb{R}^{2 n \times 2 n}, \mathbf{B} \in \mathbb{R}^{2 n \times m}$ and $\mathbf{C} \in \mathbb{R}^{p \times 2 n}(p \leq 2 n)$ are the state matrix, input matrix, and output matrix, respectively. In (4), $\mathbf{f}(t, \mathbf{x}(t)) \in$ $\mathbb{R}^{2 n}$ is the unknown nonlinear vector, representing the time-varying bounded uncertainties in the system.

The $\mathscr{L}_{1}$ adaptive control problem is formulated by dividing the control input into two parts ${ }^{25}$

$$
\mathbf{u}(t)=\mathbf{u}_{m}(t)+\mathbf{u}_{a d}(t) .
$$

In (5), $\mathbf{u}_{m}(t)=-\mathbf{K}_{m}^{T} \mathbf{x}(t)$ is the state feedback control input used to convert the system state matrix to a Hurwitz matrix with desired eigenvalues. Hence

$$
\begin{array}{r}
\dot{\mathbf{x}}(t)=\mathbf{A x}(t)+\mathbf{B}\left(-\mathbf{K}_{m}^{T} \mathbf{x}(t)+\mathbf{u}_{a d}(t)\right)+\mathbf{f}(t, \mathbf{x}(t)) \\
\left.\rightarrow \dot{\mathbf{x}}(t)=\left(\mathbf{A}-\mathbf{B} \mathbf{K}_{m}^{T}\right) \mathbf{x}(t)+\mathbf{B} \mathbf{u}_{a d}(t)\right)+\mathbf{f}(t, \mathbf{x}(t))
\end{array}
$$

In (6), the matrix $\mathbf{A}-\mathbf{B} \mathbf{K}_{m}^{T}$ is representing the desired system matrix, and is referred to as $\mathbf{A}_{m}$ from now on. As a result, the system equations in (4) can be written as

$$
\begin{aligned}
\dot{\mathbf{x}}(t) & =\mathbf{A}_{m} \mathbf{x}(t)+\mathbf{B} \mathbf{u}_{a d}(t)+\mathbf{f}(t, \mathbf{x}(t)) \\
\mathbf{y}(t) & =\mathbf{C} \mathbf{x}(t) .
\end{aligned}
$$

Remark 2. In (7), it is assumed that the initial condition of the system, $\mathbf{x}_{0}$ is bounded with the radius $\rho_{0}$

$$
\left\|\mathbf{x}_{0}\right\|_{\infty} \leq \rho_{0} \leq \infty
$$

By considering the unknown positive constant $w$ as an uncertainty on the input gain, it is assumed that the system in (7) can be rewritten as

$$
\begin{aligned}
\dot{\mathbf{x}}(t) & =\mathbf{A}_{m} \mathbf{x}(t)+\mathbf{B}\left(w \mathbf{u}_{a d}(t)+\mathbf{f}_{1}(t, \mathbf{x}(t))\right) \\
\mathbf{y}(t) & =\mathbf{C} \mathbf{x}(t) .
\end{aligned}
$$

The assumptions on how to formulate the $\mathscr{L}_{1}$ adaptive control problem are summarized in Remark $3^{25}$.

Remark 3. To formulate the $\mathscr{L}_{1}$ adaptive control problem, it is assumed

(i) Almost for all $t \geq 0$, there is a value $d_{f}$ such that $\|\mathbf{f}(t, 0)\|_{\infty} \leq d_{f}$

(ii) For any arbitrary positive value $\delta>0$, there are positive time-independent constants $d_{f_{x}}(\delta)>0$ and $d_{f_{t}}(\delta)>0$, such that for all $\|\mathbf{x}(t)\|_{\infty} \leq \delta$, the partial derivatives of $\mathbf{f}(t, \mathbf{x}(t))$ are bounded and piecewise-continuous

$$
\left\|\frac{\partial \mathbf{f}(t, \mathbf{x}(t))}{\partial \mathbf{x}}\right\|_{\infty} \leq d_{f_{x}}(\boldsymbol{\delta}),\left\|\frac{\partial \mathbf{f}(t, \mathbf{x}(t))}{\partial t}\right\|_{\infty} \leq d_{f_{t}}(\boldsymbol{\delta}) .
$$


(iii) It is assumed that the transfer zeros of the conversion matrix in (7), $\mathbf{H}(s)=\mathbf{C}\left(s \mathbf{I}-\mathbf{A}_{m}\right)^{-1} \mathbf{B}$ are all on the left-hand side of the imaginary axis.

Remark 4. Equation (9), can be converted to a semi-linear equation with some assumptions on the nonlinear function $\mathbf{f}_{1}(t, \mathbf{x}(t))$ and boundedness on the parameters as demonstrated in ${ }^{27}$. It is assumed that this function can be written as $\mathbf{f}_{1}(t, \mathbf{x}(t)) \approx$ $\theta^{T}(t)\|\mathbf{x}(t)\|_{\infty}+\sigma(t)$, with $\sigma(t)$ and $\theta^{T}(t)$ as time-varying disturbances and the time-varying uncertainty, respectively. It is assumed that $\theta(t) \in \Theta$ and $|\sigma(t)| \leq \Delta_{0}, \forall t \geq 0$. Here, $\Theta$ is a known convex compact set and $\Delta_{0}$ is a known bound for $\sigma(t)$, respectively.

Therefore, according to Remarks 3 and 4, the semi-linear structure can be written as

$$
\begin{aligned}
\dot{\mathbf{x}}(t) & =\mathbf{A}_{m} \mathbf{x}(t)+\mathbf{B}\left(w \mathbf{u}_{a d}(t)+\theta^{T}(t)\|\mathbf{x}(t)\|_{\infty}+\sigma(t)\right) \\
\mathbf{y}(t) & =\mathbf{C} \mathbf{x}(t) .
\end{aligned}
$$

\section{Standard $\mathscr{L}_{1}$ Adaptive Control Problem}

In this section, the $\mathscr{L}_{1}$ adaptive control problem and its main steps are explained. The controller is synthesized in three main steps.

\section{Step I. State predictor}

$$
\begin{aligned}
\dot{\hat{\mathbf{x}}}(t) & =\mathbf{A}_{m} \hat{\mathbf{x}}(t)+\mathbf{B}\left(\hat{w}(t) \mathbf{u}_{a d}(t)+\hat{\boldsymbol{\theta}}^{T}(t)\|\mathbf{x}(t)\|_{\infty}+\hat{\boldsymbol{\sigma}}(t)\right) \\
\hat{\mathbf{y}}(t) & =\mathbf{C} \hat{\mathbf{x}}(t),
\end{aligned}
$$

where $\hat{\theta}^{T}(t), \hat{\sigma}(t) \in \mathbb{R}^{m}$, and $\hat{w}(t)$ are the adaptive estimations for $\theta^{T}(t), \sigma(t)$, and $w$.

\section{Step II. Adaptation laws}

The adaptive estimations of $\hat{w}(t), \hat{\theta}^{T}(t)$ and $\hat{\sigma}(t)$ can be determined with the adaptation laws defined below

$$
\begin{aligned}
& \dot{\hat{\boldsymbol{\theta}}}(t)=\Gamma \operatorname{Proj}\left(\hat{\boldsymbol{\theta}}(t),-\tilde{\mathbf{x}}^{T}(t) \mathbf{P B}\|\mathbf{x}(t)\|_{\infty}\right), \hat{\boldsymbol{\theta}}(0)=\hat{\theta}_{0} \\
& \dot{\hat{\boldsymbol{\sigma}}}(t)=\Gamma \operatorname{Proj}\left(\hat{\boldsymbol{\sigma}}(t),-\tilde{\mathbf{x}}^{T}(t) \mathbf{P B}\right), \hat{\boldsymbol{\sigma}}(0)=\hat{\boldsymbol{\sigma}}_{0} \\
& \dot{\hat{w}}(t)=\Gamma \operatorname{Proj}\left(\hat{w}(t),-\tilde{\mathbf{x}}^{T}(t) \mathbf{P B} \mathbf{u}_{a d}(t)\right), \hat{w}(0)=\hat{w}_{0},
\end{aligned}
$$

where $\tilde{\mathbf{x}}(t)=\hat{\mathbf{x}}(t)-\mathbf{x}(t)$ is the state prediction error, $\Gamma \in \mathbb{R}^{+}$is the adaptation gain, and the positive definite matrix $\mathbf{P}=\mathbf{P}^{T}$ in (13), is the solution of the algebraic Lyapunov equation $\mathbf{A}_{m}^{T} \mathbf{P}+\mathbf{P} \mathbf{A}_{m}=-\mathbf{Q}$ for any arbitrary symmetric and positive definite matrix $\mathbf{Q}=\mathbf{Q}^{T}$.

Remark 5. In (13), Proj(.,.) is the projection operator that guarantees $\|\hat{\theta}(t)\|_{\infty} \leq \theta_{b},\|\hat{\sigma}(t)\|_{\infty} \leq \sigma_{b}$, and $\hat{w}(t) \in\left[w_{l}, w_{h}\right]$ with $0 \leq w_{l} \leq w_{h}$. The projection operator is defined as

$$
\begin{aligned}
& \Omega_{c}=\left\{\theta \in \mathbb{R}^{n} \mid \mathbf{f}(\theta) \leq c\right\}, 0 \leq c \leq 1 \\
& \mathbf{f}(\theta)=\frac{\left(\varepsilon_{\theta}\right) \theta^{T} \theta-\theta_{\max }^{2}}{\varepsilon_{\theta} \theta_{\max }^{2}} \\
& \operatorname{Proj}(\theta, y)= \begin{cases}y & \text { if } \mathbf{f}(\theta)<0, \\
y & \text { if } \mathbf{f}(\theta) \geq 0 \text { and } \nabla \mathbf{f}^{T} y \leq 0, \\
y-\frac{\nabla \mathbf{f}}{\|\nabla \mathbf{f}\|}\left\langle\frac{\nabla \mathbf{f}}{\|\nabla \mathbf{f}\|}, y\right\rangle \quad \text { if } \mathbf{f}(\boldsymbol{\theta}) \geq 0 \text { and } \nabla \mathbf{f}^{T} y>0,\end{cases}
\end{aligned}
$$

where $\Omega_{c}$ is a convex set with smooth bound, $\mathbf{f}(\theta)$ is a uniform convex function, $\theta_{\max }$ is the upper bound of vector $\theta$, and $\varepsilon_{\theta}$ is the projection threshold.

\section{Step III. Control law}

The control law of the adaptive feedback system is calculated according to (15)

$$
\mathbf{u}_{a d}(s)=-K \mathbf{D}(s) \hat{\eta}(s),
$$

where $\hat{\eta}(s)$ is the Laplace transform of the following signal

$$
\hat{\eta}(t)=\hat{w}(t) \mathbf{u}_{a d}(t)+\hat{\eta}_{1}(t)-\mathbf{r}_{g}(t) .
$$


In (16), $\mathbf{r}_{g}(s)=\mathbf{K}_{g} \mathbf{R}(s), \hat{\eta}_{1}(t)=\hat{\boldsymbol{\theta}}^{T}(t)\|\mathbf{x}(t)\|_{\infty}+\hat{\boldsymbol{\sigma}}(t), \mathbf{R}(s)$ is the Laplace transform of the reference input $\mathbf{r}(t)$, and the input gain $\mathbf{K}_{g}$ is defined as $\mathbf{K}_{g}=-\left(\mathbf{C A}_{m}^{-1} \mathbf{B}\right)^{-1}$. In (15), $K>0$ is the feedback gain, and $\mathbf{D}(s)$ is a diagonal matrix with elements of strictly proper transfer functions that leads to designing the low-pass filter $\mathbf{C}(s)$ in (17)

$$
\mathbf{C}(s)=w K \mathbf{D}(s)+\left(\mathbf{I}_{m}+w K \mathbf{D}(s)\right)^{-1}
$$

In (17), $\mathbf{C}(s)$ is a diagonal matrix with elements of strictly proper and stable filter with DC gain of 1.

\section{Barrier Lyapunov Function (BLF)}

Definition 1. The Barrier Lyapunov Function $(B L F)$ is a continuous, scalar and positive definite function $V_{i}(\mathbf{x})$ that has continuous first-order partial derivatives at every point in the open region $E_{i}$, containing the origin, and is the solution of the dynamic system $\dot{x}_{i}=f_{i}\left(x_{i}\right)$ on $E_{i}$. The BLF has the property of $V_{i}\left(x_{i}\right) \rightarrow \infty$ when $x_{i}$ is getting closer to the boundaries of $E_{i}$, and fulfils this condition $\exists b_{i}, V_{i}\left(x_{i}(t)\right) \leq b_{i}, \forall t \geq 0$ for any trajectory $x_{i}(t)$ starting inside $E_{i}^{40}$.

Lemma 1. Assume the dynamic system $\dot{\mathbf{z}}=\mathbf{f}(\mathbf{x}, \mathbf{w}, t)$ is smooth and has the state variables defined as $\mathbf{z}=[\mathbf{x}, \mathbf{w}]^{T}$. Let $n$ be the size of the vector $\mathbf{x}$ and $V_{i}(\mathbf{x})$ be a BLF that fulfils the condition $V_{i}\left(x_{i}\right) \rightarrow \infty$ when $x_{i} \rightarrow \pm \Delta x_{i}$, and $Q(\mathbf{w})$ be a Quadratic Lyapunov Function $(Q L F)$. Assume $V=\sum_{i=1}^{n} V_{i}\left(x_{i}\right)+Q(\mathbf{w})$, if the inequality $\dot{V}=\left[\frac{\partial V}{\partial \mathbf{z}}\right]^{T} \mathbf{f} \leq 0$, holds all over the set $S=\left\{(\mathbf{x}, \mathbf{w}):\left|x_{i}\right|<\Delta x_{i}\right\}$, then any trajectory, satisfying the initial constraints $\left|x_{i}(0)\right|<\Delta x_{i}, \forall i$ will remain inside $S$ for all times.

Remark 6. In Lemma 1, the states are split into the constrained states $\mathbf{x}$ and unconstrained states $\mathbf{w}$. In this paper, BLF is chosen from ${ }^{41}$ and is defined as in (18)

$$
V(x)=\frac{1}{2 D} \tan \left(D x^{2}\right),
$$

where $D=\frac{\pi}{\Delta^{2}}$.

\section{The Proposed Control Structure}

In this section, we merge the concepts of BLF and adaptive backstepping method to develop a new scheme for $\mathscr{L}_{1}$ adaptive controller. The aim of the proposed controller is to eliminate the filter $C(s)$ in the design process of $\mathscr{L}_{1}$ adaptive approach through a novel formulation and structure for the adaptation law and control input. In this way, it is possible to keep the value of adaptive gain high and unchanged compared to the case when the filter exists. As stated $\mathrm{in}^{27}$, designing such a filter is very difficult and even impossible in some cases. It is worth noting that, the performance of $\mathscr{L}_{1}$ adaptive control is heavily dependent on this filter design, and in case that a suitable filter is not designed, the $\mathscr{L}_{1}$ adaptive controller is practically inefficient. Therefore, in the new design by eliminating this filter, we still keep the robustness and stability performance by having a high adaptive gain. In the following, the structure of the new design for the $\mathscr{L}_{1}$ adaptive controller with BLF is described.

\section{Problem Formulation}

The problem structure proposed in this section can be applied to the systems with the position and velocity as the states. Therefore, it covers a wide range of dynamical systems, including the Euler-Lagrange robotic systems. By starting from the state-space dynamic model represented in (4) and separating the position and velocity states, the state-space model of the system can be reformulated as

$$
\begin{aligned}
& \dot{\mathbf{x}}_{1}(t)=\mathbf{v}_{1}(t) \\
& \ddot{\mathbf{x}}_{1}(t)=\dot{\mathbf{v}}_{1}(t)=\mathbf{A}^{\prime} \mathbf{v}_{1}(t)+\mathbf{b u}(t)+\mathbf{f}\left(\mathbf{x}_{1}(t), \mathbf{v}_{1}(t), t\right) \\
& \mathbf{y}(t)=\left[\mathbf{0}_{\mathbf{p} \times \mathbf{n}} \mathbf{c}\right] \mathbf{x}(t) .
\end{aligned}
$$

In (19), $\mathbf{x}_{1}(t)=\left[q_{1}, q_{2}, \ldots, q_{n}\right]^{T}$ and $\mathbf{v}_{1}(t)=\left[\dot{q}_{1}, \dot{q}_{2}, \ldots, \dot{q}_{n}\right]^{T}$ are the position and velocity vectors, respectively. Also, $\mathbf{x}(t)=$ $\left[\mathbf{x}_{1}^{T}(t) \mathbf{v}_{1}^{T}(t)\right]^{T}$ is the full state vector of the system. The matrices $\mathbf{A}^{\prime} \in \mathbb{R}^{n \times n}, \mathbf{b} \in \mathbb{R}^{n \times m}, \mathbf{c} \in \mathbb{R}^{p \times n}$ in (19), and the rest of the parameters are defined as before in (4). By writing (19) in the state space form, the matrices $\mathbf{A}, \mathbf{B}$ and $\mathbf{C}$ in (4) can be defined as

$$
\mathbf{A}=\left[\begin{array}{cc}
\mathbf{0}_{n \times n} & \mathbf{I}_{n \times n} \\
\mathbf{0}_{n \times n} & \mathbf{A}^{\prime}
\end{array}\right], \mathbf{B}=\left[\begin{array}{c}
\mathbf{0}_{n \times m} \\
\mathbf{b}
\end{array}\right], \mathbf{C}=\left[\begin{array}{ll}
\mathbf{0}_{p \times n} & \mathbf{c}
\end{array}\right] .
$$


By splitting the control law into two parts

$$
\mathbf{u}(t)=\mathbf{u}_{m}(t)+\varpi \mathbf{u}_{a d}(t),
$$

we define $\varpi \in \mathbb{R}^{+}, \varpi \in\left[\varpi_{l}, \varpi_{u}\right]$ (with $0<\varpi_{l}<\varpi_{u}$ ) as the uncertainty on the input gain. By applying the velocity feedback $\mathbf{u}_{m}(t)=-\mathbf{K}_{m}^{T} \mathbf{v}_{1}(t)$, the second equation in (19) is converted to a system with a Hurwitz state matrix as below

$$
\begin{aligned}
& \dot{\mathbf{x}}_{1}(t)=\mathbf{v}_{1}(t) \\
& \dot{\mathbf{v}}_{1}(t)=\mathbf{A}_{m}^{\prime} \mathbf{v}_{1}(t)+\mathbf{b}\left(\varpi \mathbf{u}_{a d}(t)+\mathbf{f}_{1}\left(\mathbf{x}_{1}(t), \mathbf{v}_{1}(t), t\right)\right),
\end{aligned}
$$

Remark 7. According to Remarks 3 and $4, \mathbf{f}_{1}\left(\mathbf{x}_{1}(t), \mathbf{v}_{1}(t), t\right)$ in (22) can be considered as $\theta_{1}^{T}(t)\|\mathbf{x}(t)\|_{\infty}+\sigma_{1}(t)$ and the nonlinear system can be transformed into a semi-linear system. Therefore, the system dynamic and the state observer dynamic can be written as

$$
\begin{aligned}
& \dot{\mathbf{x}}_{1}(t)=\mathbf{v}_{1}(t) \\
& \dot{\mathbf{v}}_{1}(t)=\mathbf{A}_{m}^{\prime} \mathbf{v}_{1}(t)+\mathbf{b}\left(\varpi \mathbf{u}_{a d}(t)+\theta_{1}^{T}(t)\|\mathbf{x}(t)\|_{\infty}+\sigma_{1}(t)\right) \\
& \mathbf{y}(t)=\mathbf{C} \mathbf{x}(t), \\
& \dot{\hat{\mathbf{x}}}_{1}(t)=\hat{\mathbf{v}}_{1}(t) \\
& \dot{\hat{\mathbf{v}}}_{1}(t)=\mathbf{A}_{m}^{\prime} \hat{\mathbf{v}}_{1}(t)+\mathbf{b}\left(\hat{\boldsymbol{\omega}} \mathbf{u}_{a d}(t)+\hat{\theta}_{1}^{T}(t)\|\mathbf{x}(t)\|_{\infty}+\hat{\sigma}_{1}(t)\right) \\
& \hat{\mathbf{y}}(t)=\mathbf{C} \hat{\mathbf{x}}(t) .
\end{aligned}
$$

\section{$\mathscr{L}_{1}$ Adaptive Back-Stepping Controller with BLF and Position-Velocity Constraints}

The primary aim to design the novel adaptive controller is to ensure that the system position and velocity follow a bounded and smooth desired trajectory. We assume that the position estimation vector $\hat{\mathbf{x}}_{1}(t)$ and its derivative in (24) are upper-bounded as

$$
\left\|\hat{\mathbf{x}}_{1}(t)\right\|_{\infty} \leq x_{\max } .
$$

By defining the position tracking error as

$$
\mathbf{e}_{x}(t)=\hat{\mathbf{x}}_{1}(t)-\mathbf{x}_{1}=\tilde{\mathbf{x}}_{1}(t),
$$

it is assumed that this error is constrained at all times, including the initial condition, by a predetermined bound as defined in (27)

$$
\left\|\mathbf{e}_{x}(t)\right\|_{\infty}<\Delta e_{x},\left\|\mathbf{e}_{x}(0)\right\|_{\infty}<\Delta e_{x} .
$$

Remark 8. According to (26), it can be readily concluded that the position is bounded with a specific upper bound as in (28)

$$
\begin{aligned}
& \mathbf{x}_{1}(t)=\hat{\mathbf{x}}_{1}(t)-\mathbf{e}_{x}(t) \rightarrow\left\|\mathbf{x}_{1}(t)\right\|_{\infty}=\left\|\hat{\mathbf{x}}_{1}(t)-\mathbf{e}_{x}(t)\right\|_{\infty} \leq \\
& \left\|\hat{\mathbf{x}}_{1}(t)\right\|_{\infty}+\left\|\mathbf{e}_{x}(t)\right\|_{\infty} \leq x_{\max }+\Delta e_{x}=\Delta x \rightarrow\left\|\mathbf{x}_{1}(t)\right\|_{\infty} \leq \Delta x .
\end{aligned}
$$

By multiplying both sides of the velocity equation in (23) with the parameter $\mu$, it is possible to eliminate the input control gain as follows

$$
\mu \dot{\mathbf{v}}_{1}(t)=\mu \mathbf{A}_{m}^{\prime} \mathbf{v}_{1}(t)+\mathbf{u}_{a d}(t)+\theta^{T}(t)\|\mathbf{x}(t)\|_{\infty}+\sigma(t),
$$

where $\mu=\mathbf{B}_{m}^{-1} \varpi \mathbf{b}^{T}, \mathbf{B}_{m}=\varpi \mathbf{b}^{T} \mathbf{b} \varpi, \theta^{T}(t)=\varpi^{-1} \theta_{1}^{T}(t)$, and $\sigma(t)=\varpi^{-1} \sigma_{1}(t)$.

The adaptive backstepping scheme is introduced, by defining the velocity error equation as

$$
\dot{\mathbf{e}}_{x}(t)=\dot{\hat{\mathbf{x}}}_{1}(t)-\dot{\mathbf{x}}_{1}(t)=\dot{\hat{\mathbf{x}}}_{1}(t)-\mathbf{v}_{1}(t) .
$$

Assuming that $\mathbf{v}_{d}$ is the desired velocity, the velocity tracking error $\mathbf{e}_{v}=\mathbf{v}_{d}-\mathbf{v}_{1}$ should be designed in a way that to ensure the convergence of the position tracking error $\mathbf{e}_{x}$ defined in (26). Similarly, we define the following constraint on the velocity tracking error at all times (including the initial condition $\left\|\mathbf{e}_{v}(0)\right\|<\Delta e_{v}$ ).

$$
\left\|\mathbf{e}_{v}(t)\right\|<\Delta e_{v}
$$


As mentioned in Remark 6, the first step is to define the BLF as

$$
\mathrm{V}_{1}(t)=\frac{1}{2 \Lambda} \tan \left(\Lambda \mathbf{e}_{x}^{T}(t) \mathbf{e}_{x}(t)\right), \Lambda=\frac{\pi}{2 \Delta e_{x}^{2}}
$$

the tracking error dynamic is defined as

$$
\dot{\mathbf{e}}_{x}(t)=\dot{\hat{\mathbf{x}}}_{1}(t)+\mathbf{e}_{v}(t)-\mathbf{v}_{d}(t)
$$

By calculating the derivative of $\mathrm{V}_{1}(\mathrm{t})$ in (32)

$$
\dot{\mathrm{V}}_{1}(t)=\mathbf{e}_{x}^{T}(t) \dot{\mathbf{e}}_{x}(t)\left(1+\tan ^{2}\left(\Lambda \mathbf{e}_{x}^{T}(t) \mathbf{e}_{x}(t)\right)\right),
$$

and with substituting (33) in (34), it can be rewritten as

$$
\dot{\mathrm{V}}_{1}(t)=\mathbf{e}_{x}^{T}(t)\left(\dot{\hat{\mathbf{x}}}_{1}(t)+\mathbf{e}_{v}(t)-\mathbf{v}_{d}(t)\right)\left(1+\tan ^{2}\left(\Lambda \mathbf{e}_{x}^{T}(t) \mathbf{e}_{x}(t)\right)\right) .
$$

Remark 9. Considering (35), two appropriate choices for $\mathbf{v}_{d}(t)$ is proposed below

1. Selecting $\mathbf{v}_{d}(t)$ as a nonlinear function

$$
\mathbf{v}_{d}(t)=\dot{\hat{\mathbf{x}}}_{1}(t)+\frac{k_{x} \mathbf{e}_{x}(t)}{\left(1+\tan ^{2}\left(\Lambda \mathbf{e}_{x}^{T}(t) \mathbf{e}_{x}(t)\right)\right)} \rightarrow \dot{V}_{1}(t)=-k_{x} \mathbf{e}_{x}^{T}(t) \mathbf{e}_{x}(t)+\mathbf{e}_{x}^{T}(t) \mathbf{e}_{v}(t)\left(1+\tan ^{2}\left(\Lambda \mathbf{e}_{x}^{T}(t) \mathbf{e}_{x}(t)\right)\right) .
$$

2. Selecting $\mathbf{v}_{d}(t)$ as a linear function

$$
\mathbf{v}_{d}(t)=\dot{\hat{\mathbf{x}}}_{1}(t)+k_{x} \mathbf{e}_{x}(t) \rightarrow \dot{V}_{1}(t)=\left(-k_{x} \mathbf{e}_{x}^{T}(t) \mathbf{e}_{x}(t)+\mathbf{e}_{x}^{T}(t) \mathbf{e}_{v}(t)\right)\left(1+\tan ^{2}\left(\Lambda \mathbf{e}_{x}^{T}(t) \mathbf{e}_{x}(t)\right)\right),
$$

where $k_{x}>0$ is the design parameter. Since the term $\frac{k_{x}}{\left(1+\tan ^{2}\left(\Lambda \mathbf{e}_{x}^{T}(t) \mathbf{e}_{x}(t)\right)\right)}$ tends to zero if $\left\|\mathbf{e}_{x}(t)\right\|_{\infty} \rightarrow \Delta e_{x}$, the nonlinear option for $\mathbf{v}_{d}(t)$ is excluded in the subsequent analyses.

Remark 10. According to (37) and definition of the velocity error, $\mathbf{e}_{v}(t)=\mathbf{v}_{d}(t)-\mathbf{v}_{1}(t)$, the stability is guaranteed, i.e. $\dot{V}_{1}(t) \leq$ 0 , if $\mathbf{v}_{1}(t)=\mathbf{v}_{d}(t)$.

Therefore, the velocity error $\mathbf{e}_{v}(t)$ used for the second step of the back-stepping method can be written as

$$
\dot{\mathbf{e}}_{v}(t)=\dot{\mathbf{v}}_{d}(t)-\dot{\mathbf{v}}_{1}(t) \rightarrow \mu \dot{\mathbf{e}}_{v}(t)=\mu \dot{\mathbf{v}}_{d}(t)-\mu \dot{\mathbf{v}}_{1}(t)=\mu \dot{\mathbf{v}}_{d}(t)-\mu \mathbf{A}_{m}^{\prime} \mathbf{v}_{1}(t)-\mathbf{u}_{a d}(t)-\theta^{T}(t)\|\mathbf{x}(t)\|_{\infty}-\sigma(t)
$$

After some mathematical manipulation, (38) can be rewritten as

$$
\mu \dot{\mathbf{e}}_{v}(t)=-\mathbf{u}_{a d}(t)-\theta^{\mathrm{T}}(t)\|\mathbf{x}(t)\|_{\infty}-\sigma(t)+\mu \mathbf{A}_{1}^{T} \xi(t),
$$

where $\mathbf{A}_{1}^{T}=\left[\mathbf{I}_{n},-\mathbf{A}_{m}^{\prime}\right], \xi(t)=\left[\begin{array}{c}\dot{\mathbf{v}}_{d}(t) \\ \mathbf{v}_{1}(t)\end{array}\right]$. On the other hand, $\xi(t)$ and $\mathbf{A}_{1}^{T}$ are known values while $\mu, \theta^{T}(t)$, and $\sigma(t)$ are unknown values. Therefore, they are replaced with their estimated values $\hat{\mu}, \hat{\theta}^{T}(t)$, and $\hat{\sigma}(t)$, achieved through the adaptive algorithm. According to (37), the derivative of the velocity $\mathbf{v}_{d}(t)$ can be written as

$$
\dot{\mathbf{v}}_{d}(t)=\ddot{\hat{\mathbf{x}}}_{1}(t)+k_{x} \dot{\mathbf{e}}_{x}(t)=\ddot{\hat{\mathbf{x}}}_{1}(t)+k_{x}\left(\mathbf{e}_{v}(t)-k_{x} \mathbf{e}_{x}(t)\right) \text {. }
$$

To derive the control signal and adaptation laws as the next step, the following Lyapunov function is proposed

$$
\mathrm{V}_{2}(t)=\mathrm{V}_{1}(t)+\frac{\mu}{2 \digamma} \tan \left(\digamma \mathbf{e}_{v}^{T}(t) \mathbf{e}_{v}(t)\right)+\frac{1}{2} \tilde{u}^{T} \Gamma^{-1} \tilde{\mu}+\frac{1}{2} \tilde{\theta}^{T} \Gamma^{-1} \tilde{\theta}+\frac{1}{2} \tilde{\sigma}^{T} \Gamma^{-1} \tilde{\sigma}, \digamma=\frac{\pi}{2 \Delta e_{v}^{2}}
$$

In (41), $\tilde{\mu}(t)=\mu-\hat{\mu}(t), \tilde{\theta}(t)=\theta(t)-\hat{\theta}(t)$, and $\tilde{\sigma}(t)=\sigma(t)-\hat{\sigma}(t)$ are the adaptation errors; $\mathrm{V}_{1}(t)$ is the BLF described in (32), $\Gamma \in \mathbb{R}^{+}$is the adaptation gain, and $\Delta e_{v}$ is the constraint applied to the velocity error $\mathbf{e}_{v}$.

Remark 11. The constraints applied to the position error may cause rapid changes near the boundary with unacceptable velocity values. Therefore, it is necessary to consider the second Lyapunov function as a BLF. 
In this case, the derivative of $\mathrm{V}_{2}(t)$ in (41) can be written as

$$
\dot{\mathrm{V}}_{2}(t)=\dot{\mathrm{V}}_{1}(t)+\mu \mathbf{e}_{v}^{T}(t)\left(1+\tan ^{2}\left(\digamma \mathbf{e}_{v}^{T}(t) \mathbf{e}_{v}(t)\right)\right) \dot{\mathbf{e}}_{v}(t)+\tilde{\mu}^{T}(t) \Gamma^{-1} \dot{\tilde{\mu}}(t)+\tilde{\theta}^{T}(t) \Gamma^{-1} \dot{\tilde{\theta}}(t)+\tilde{\sigma}^{T}(t) \Gamma^{-1} \dot{\tilde{\sigma}}(t) .
$$

Substituting (37) and (39) into (42) yields

$$
\begin{aligned}
& \dot{\mathrm{V}}_{2}(t)=\left(-k_{x} \mathbf{e}_{x}^{T}(t) \mathbf{e}_{x}(t)+\mathbf{e}_{x}^{T}(t) \mathbf{e}_{v}(t)\right)\left(1+\tan ^{2}\left(\Lambda \mathbf{e}_{x}^{T}(t) \mathbf{e}_{x}(t)\right)\right)+\mathbf{e}_{v}^{T}(t)\left(1+\tan ^{2}\left(\digamma \mathbf{e}_{v}^{T}(t) \mathbf{e}_{v}(t)\right)\right) \times \\
& \left(-\mathbf{u}_{a d}(t)-\theta^{T}(t)\|\mathbf{x}(t)\|_{\infty}-\sigma(t)+\mu(t) \mathbf{A}_{1}^{T} \xi(t)\right)+\tilde{\mu}^{T}(t) \Gamma^{-1} \dot{\tilde{\mu}}(t)+\tilde{\theta}^{T}(t) \Gamma^{-1} \dot{\tilde{\theta}}(t)+\tilde{\sigma}^{T}(t) \Gamma^{-1} \dot{\tilde{\sigma}}(t) .
\end{aligned}
$$

By designing the control input signal $\mathbf{u}_{a d}(t)$ in (44), it is possible to cancel the extra terms in (43) so that $\dot{\mathrm{V}}_{2}(t) \leq 0$ is satisfied

$$
\mathbf{u}_{a d}(t)=-\hat{\theta}^{T}(t)\|\mathbf{x}(t)\|_{\infty}-\hat{\sigma}(t)+\hat{\mu}(t) \mathbf{A}_{1}^{T} \xi(t)+\mathbf{e}_{x}(t) \frac{\left(1+\tan ^{2}\left(\Lambda \mathbf{e}_{x}^{T}(t) \mathbf{e}_{x}(t)\right)\right)}{\left(1+\tan ^{2}\left(\digamma \mathbf{e}_{v}^{T}(t) \mathbf{e}_{v}(t)\right)\right)}+\mathbf{k}_{v} \mathbf{e}_{v}(t)+\mathbf{K}_{g} \mathbf{r}(t)
$$

In (44), $\mathbf{k}_{v} \in \mathbb{R}^{n \times n}$ is a diagonal and positive definite matrix which must be designed. $\mathbf{r}(t)$ and $\mathbf{K}_{g}=-\left(\mathbf{c}\left(\mathbf{A}_{m}^{\prime}\right)^{-1} \mathbf{b}\right)^{-1}$ are the reference trajectory and the feed-forward gain, respectively. Such a control input causes the velocity tracking error in (39) to be

$$
\mu \dot{\mathbf{e}}_{v}(t)=-\mathbf{k}_{v} \mathbf{e}_{v}(t)-\mathbf{e}_{x}(t) \frac{\left(1+\tan ^{2}\left(\Lambda \mathbf{e}_{x}^{T}(t) \mathbf{e}_{x}(t)\right)\right)}{\left(1+\tan ^{2}\left(\digamma \mathbf{e}_{v}^{T}(t) \mathbf{e}_{v}(t)\right)\right)}-\tilde{\theta}^{T}(t)\|\mathbf{x}(t)\|_{\infty}-\tilde{\sigma}(t)+\tilde{\mu}(t) \mathbf{A}_{1}^{T} \xi(t)-\mathbf{K}_{g} \mathbf{r}(t) .
$$

Substituting (44) into (43) yields

$$
\begin{aligned}
& \dot{\mathrm{V}}_{2}(t)=-k_{x} \mathbf{e}_{x}^{T}(t) \mathbf{e}_{x}(t)\left(1+\tan ^{2}\left(\Lambda \mathbf{e}_{x}^{T}(t) \mathbf{e}_{x}(t)\right)\right)-\mathbf{k}_{v} \mathbf{e}_{v}^{T}(t) \mathbf{e}_{v}(t)\left(1+\tan ^{2}\left(\digamma \mathbf{e}_{v}^{T}(t) \mathbf{e}_{v}(t)\right)\right)+ \\
& \tilde{\mu}(t)\left(\mathbf{e}_{v}^{T}(t)\left(1+\tan ^{2}\left(\digamma \mathbf{e}_{v}^{T}(t) \mathbf{e}_{v}(t)\right)\right) \mathbf{A}_{1}^{T} \xi(t)+\Gamma^{-1} \dot{\tilde{\mu}}(t)\right)+\tilde{\theta}^{T}(t)\left(-\mathbf{e}_{v}^{T}(t)\left(1+\tan ^{2}\left(\digamma \mathbf{e}_{v}^{T}(t) \mathbf{e}_{v}(t)\right)\right)\|\mathbf{x}(t)\|_{\infty}+\Gamma^{-1} \tilde{\boldsymbol{\theta}}(t)\right) \\
& -\tilde{\sigma}^{T}(t)\left(\left(1+\tan ^{2}\left(\digamma \mathbf{e}_{v}^{T}(t) \mathbf{e}_{v}(t)\right)\right) \mathbf{e}_{v}(t)+\Gamma^{-1} \tilde{\tilde{\boldsymbol{\sigma}}}(t)\right)-\mathbf{e}_{v}^{T}(t) \mathbf{K}_{g} \mathbf{r}(t)\left(1+\tan ^{2}\left(\digamma \mathbf{e}_{v}^{T}(t) \mathbf{e}_{v}(t)\right)\right) .
\end{aligned}
$$

Since $\dot{\tilde{\mu}}(t)=-\dot{\hat{\mu}}(t), \dot{\tilde{\theta}}(t)=-\dot{\hat{\theta}}(t)$, and $\dot{\tilde{\sigma}}(t)=-\dot{\hat{\sigma}}(t)$, and to guarantee the non-positiveness of $\dot{\mathrm{V}}_{2}(t)$, the following adaptation laws are proposed to remove the undesirable components in (46)

$$
\begin{aligned}
& \dot{\hat{\mu}}(t)=\Gamma \operatorname{Proj}\left(\hat{\mu}(t), \mathbf{e}_{v}^{T}(t)\left(1+\tan ^{2}\left(\digamma \mathbf{e}_{v}^{T}(t) \mathbf{e}_{v}(t)\right)\right) \mathbf{A}_{1}^{T} \xi(t)\right), \hat{\mu}(0)=\hat{\mu}_{0} \\
& \dot{\hat{\theta}}(t)=-\Gamma \operatorname{Proj}\left(\hat{\boldsymbol{\theta}}(t), \mathbf{e}_{v}^{T}(t)\left(1+\tan ^{2}\left(\digamma \mathbf{e}_{v}^{T}(t) \mathbf{e}_{v}(t)\right)\right)\|\mathbf{x}(t)\|_{\infty}\right), \hat{\boldsymbol{\theta}}(0)=\hat{\theta}_{0} \\
& \dot{\hat{\sigma}}(t)=-\Gamma \operatorname{Proj}\left(\hat{\sigma}(t),\left(1+\tan ^{2}\left(\digamma \mathbf{e}_{v}^{T}(t) \mathbf{e}_{v}(t)\right)\right) \mathbf{e}_{v}(t)\right), \hat{\sigma}(0)=\hat{\sigma}_{0} .
\end{aligned}
$$

By merging (46) and (47), the right-hand side in (46) can be simplified as

$$
\dot{\mathrm{V}}_{2}(t) \leq-k_{x} \mathbf{e}_{x}^{T}(t) \mathbf{e}_{x}(t)\left(1+\tan ^{2}\left(\Lambda \mathbf{e}_{x}^{T}(t) \mathbf{e}_{x}(t)\right)\right)-\mathbf{e}_{v}^{T}(t)\left(\mathbf{K}_{g} \mathbf{r}(t)+\mathbf{k}_{v} \mathbf{e}_{v}(t)\right)\left(1+\tan ^{2}\left(\digamma \mathbf{e}_{v}^{T}(t) \mathbf{e}_{v}(t)\right)\right) .
$$

Assuming that $\left\|\mathbf{K}_{g} \mathbf{r}(t)\right\|_{\infty} \leq \bar{k}_{g}$ and $\mathbf{k}_{v}$ is designed so that $\mathbf{k}_{v}=k_{v}^{\prime} \mathbf{I}_{n}, k_{v}^{\prime} \geq 10 \bar{k}_{g}$. By knowing the upper bounds for the errors from (27) and (31), the right hand-side of (48) can be written as

$$
\begin{aligned}
& \dot{\mathrm{V}}_{2}(t) \leq-k_{x} \Delta e_{x}^{2}\left(1+\tan ^{2}\left(\Lambda \mathbf{e}_{x}^{T}(t) \mathbf{e}_{x}(t)\right)\right)-\left(\left\|\mathbf{K}_{g} \mathbf{r}(t)\right\|_{\infty} \Delta e_{v}+k_{v}^{\prime} \Delta e_{v}{ }^{2}\right)\left(1+\tan ^{2}\left(\digamma \mathbf{e}_{v}^{T}(t) \mathbf{e}_{v}(t)\right)\right) \\
& \dot{\mathrm{V}}_{2}(t) \leq-k_{x} \Delta e_{x}^{2}\left(1+\tan ^{2}\left(\Lambda \mathbf{e}_{x}^{T}(t) \mathbf{e}_{x}(t)\right)\right)-\left(\bar{k}_{g} \Delta e_{v}+k_{v}^{\prime} \Delta e_{v}{ }^{2}\right)\left(1+\tan ^{2}\left(\digamma \mathbf{e}_{v}^{T}(t) \mathbf{e}_{v}(t)\right)\right) .
\end{aligned}
$$

Therefore, it is possible to conclude that the derivative of $\mathrm{V}_{2}(t)$ is negative over the set $S$ as follows

$$
\dot{\mathrm{V}}_{2}(t) \leq 0 \rightarrow S=\left\{\left(\mathbf{e}_{x}(t), \mathbf{e}_{v}(t), \tilde{\mu}(t), \tilde{\theta}(t), \tilde{\sigma}(t)\right):\left\|\mathbf{e}_{x}(t)\right\|_{\infty}<\Delta e_{x},\left\|\mathbf{e}_{v}(t)\right\|_{\infty}<\Delta e_{v}\right\} .
$$

The main properties of the proposed technique can be formulated as in Theorem 1 presented below.

Theorem 1. For the dynamic system defined in (19) with the Lyapunov function (41), the control and adaptation laws given by (44) and (47). Assuming that the initial conditions are satisfying

$$
\left\|\mathbf{e}_{x}(0)\right\|_{\infty}<\Delta e_{x},\left\|\mathbf{e}_{v}(0)\right\|_{\infty}<\Delta e_{v}
$$

the following properties will be true for the system trajectories 
(i) The tracking errors $\mathbf{e}_{x}(t)$ and $\mathbf{e}_{v}(t)$ remain inside a compact set, and the system state variables satisfy the constraints $\left\|\mathbf{e}_{x}(t)\right\|_{\infty}<\Delta e_{x},\left\|\mathbf{e}_{v}(t)\right\|_{\infty}<\Delta e_{v}$ for all times $t$.

(ii) All signals in the closed-loop system are bounded.

(iii) The position and velocity tracking errors $\left(\mathbf{e}_{x}(t), \mathbf{e}_{v}(t)\right)$ tend to zero asymptotically.

Proof.

(i) Given that $\mathrm{V}_{2}(0)$ is bounded and $\dot{\mathrm{V}}_{2}(t) \leq 0$, it can be readily concluded that $\mathrm{V}_{2}(t)<\mathrm{V}_{2}(0)$ along the system trajectories. Therefore, according to Lemma 1 , for any system trajectories satisfying (51), $\left\|\mathbf{e}_{x}(t)\right\|_{\infty}<\Delta e_{x},\left\|\mathbf{e}_{v}(t)\right\|_{\infty}<\Delta e_{v}$.

(ii) According to (i) in Theorem $1, \mathbf{e}_{x}(t)$ and $\mathbf{e}_{v}(t)$ are bounded for all times. Following (38), and assuming that $\mathbf{v}_{d}(t)$ and $\dot{\mathbf{v}}_{d}(t)$ are bounded, it can be concluded that $\xi(t)$ in (39) is bounded. Moreover, by looking at (47) and Remark 4, the estimated values for the uncertainty $\hat{\mu}(t), \hat{\theta}(t), \hat{\sigma}(t)$, and the adaptation errors $\tilde{\mu}(t), \tilde{\theta}(t), \tilde{\sigma}(t)$ are bounded. From (44), it can also be seen that the control law $\mathbf{u}_{a d}(t)$ will remain bounded.

(iii) According to (50) and the previous results, it is determined that $\ddot{\mathrm{V}}_{2}(t)$ is bounded. Therefore, applying the Barbalats lemma ${ }^{42}$ yields that the tracking errors $\mathbf{e}_{x}(t)$ and $\mathbf{e}_{v}(t)$ are asymptotically converging to zero.

Remark 12. It is worthwhile to note that the initial conditions $\left\|\mathbf{e}_{x}(0)\right\|_{\infty}<\Delta e_{x},\left\|\mathbf{e}_{v}(0)\right\|_{\infty}<\Delta e_{v}$ imply that $k_{x}$ and $\Delta e_{v}$ cannot be chosen independent from each other. Therefore, $\Delta e_{v}$ must be chosen in a way that the following inequality is satisfied

$$
\mathbf{e}_{v}(0)=\dot{\mathbf{x}}_{1}(0)+k_{x} \mathbf{e}_{x}(0)-\mathbf{v}_{1}(0) \rightarrow\left\|\dot{\mathbf{x}}_{1}(0)+k_{x} \mathbf{e}_{x}(0)-\mathbf{v}_{1}(0)\right\|_{\infty} \leq\left\|\dot{\mathbf{x}}_{1}(0)-\mathbf{v}_{1}(0)\right\|_{\infty}+k_{x} \Delta e_{x}<\Delta e_{v} .
$$

Similarly, according to (28), the value of $\left\|\mathbf{x}_{1}(t)\right\|_{\infty}$ is bounded due to the upper value of $\Delta e_{x}$. The choice of $\Delta e_{x}$ also provides a constraint for the maximum velocity as follows

$$
\|\mathbf{v}(t)\|_{\infty} \leq\left\|\mathbf{x}_{d}(t)+k_{x} \mathbf{e}_{x}(t)\right\|_{\infty}+\left\|\mathbf{e}_{v}(t)\right\|_{\infty} \leq \max _{t>0}\left|\mathbf{x}_{d}(t)\right|+k_{x} \Delta e_{x}+\Delta e_{v}=\Delta v
$$
2.

In order to gain a better understanding, the schematic block diagram of the proposed control system is illustrated in Figure

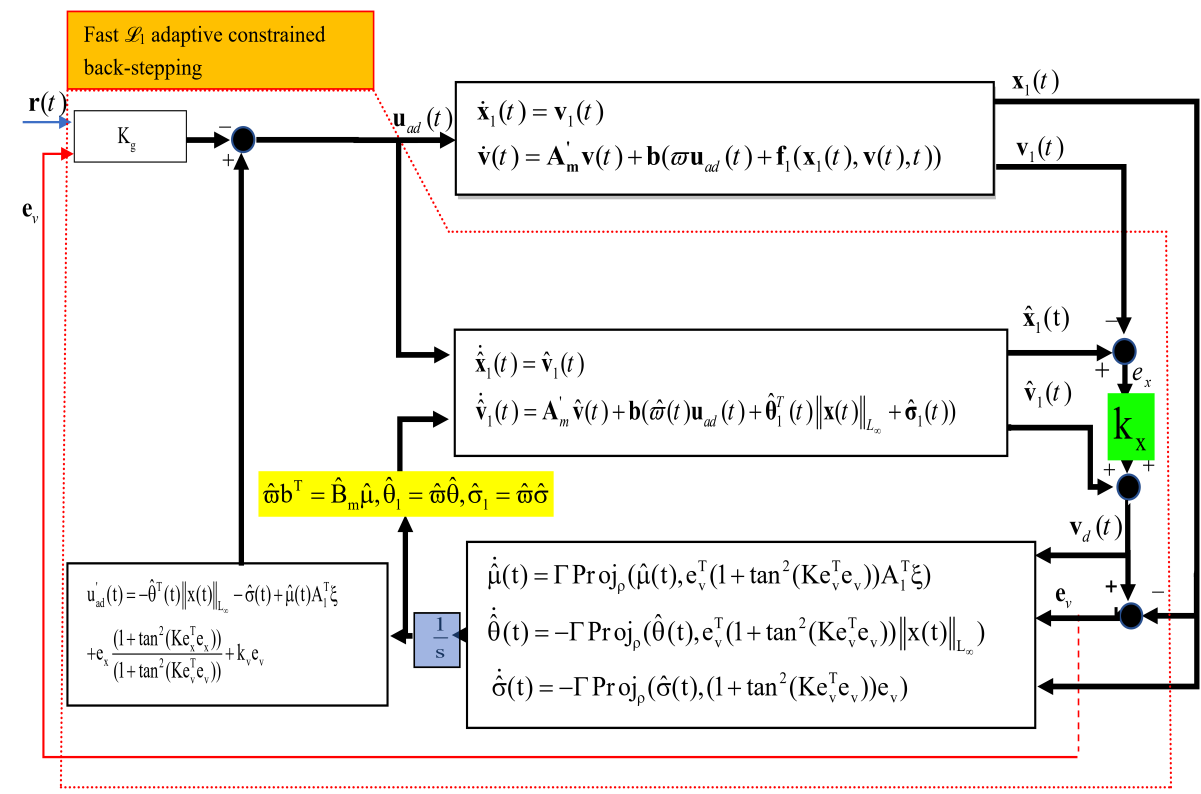

Figure 2. The schematic block diagram illustrating the details of the proposed scheme. 


\section{Simulation Results}

The performance of the proposed $\mathscr{L}_{1}$ adaptive backstepping controller with BLF is studied here under two different scenarios with time-varying uncertainties and disturbances. In both cases, the joint position and velocity constraints are considered in the design. In the first scenario the tracking performance of a single-link robotic manipulator is evaluated for the new design, while in the second scenario the more complex problem of controlling a 6-DOF ROV robot is investigated. In both cases, the tracking errors are compared based on these two measures

(a) The Integral Absolute Error (IAE). i.e. IAE $=\int_{0}^{s t} e(t) \mathrm{d} t^{36}$

(b) Integral Error with Trapezoidal Rule (IETR) ${ }^{43}$ :

$$
\operatorname{IETR}=\sum_{t=0}^{s t-b}\left(\int_{t}^{t+b} e(t) \mathrm{d} t\right) \approx \sum_{t=0}^{s t-b}\left(b\left[\frac{e(t)+e(t+b)}{2}\right]\right)
$$

where $s t$ in the equations above represents the simulation time and $b$ is the time step.

\section{Case study I}

The dynamic of a single-link robot can be described as $^{25}$

$$
J \ddot{\phi}(t)=u(t)+\frac{M g l \cos (\phi(t))}{2}+G(t)+D(t) \phi(t)+F(t) \dot{\phi}(t) .
$$

In (54), $\ddot{\phi}(t), \dot{\phi}(t)$ and $\phi(t)$ are the manipulator angular acceleration, velocity, and position, respectively. Here, $u(t)$ is the control effort, $J$ is the unknown moment of inertia for the robot, $M$ is the unknown link mass, and $l$ is the link length. Moreover, $D(t)$ and $F(t)$ are the position-dependent external torque coefcient and unknown time-varying friction coefcient, respectively. Moreover, $\sigma(t)$ is a time-varying unknown and bounded disturbance and we have the following assumptions for the controller design

$$
\begin{aligned}
& \mathbf{A}_{m}=\left[\begin{array}{cc}
0 & 1 \\
-1 & -1.4
\end{array}\right], \mathbf{b}=\left[\begin{array}{l}
1 \\
0
\end{array}\right] \\
& \theta(t)=\left[\begin{array}{ll}
1+\frac{D(t)}{J} & 1.4+\frac{F(t)}{J}
\end{array}\right]^{T}=\left[\begin{array}{ll}
\sin (0.5 \pi t)+\cos (\pi t) & -1+0.1 \sin (3 \pi t)
\end{array}\right]^{T} \\
& \sigma_{1}(t)=\frac{M g l \cos (\phi(t))}{2 J}+\frac{\sigma(t)}{J}=\cos (\phi(t))+2 \sin (\pi t)+\cos \left(\frac{7 \pi}{5} t\right) \\
& w=1 / J=1.5 .
\end{aligned}
$$

The numerical results showing the performance of the proposed adaptive controller compared to the standard $\mathscr{L}_{1}$ adaptive control are plotted in Figure 3. As can be seen from the figure, the tracking error is converging to zero and the estimated and true states of the system are demonstrating a close match. The results confirm the capability of the proposed controller in removing the time-varying disturbances and uncertainties, and demonstrates a good tracking performance. The performance of the proposed controller is compared numerically against the standard $\mathscr{L}_{1}$ controller in Table 1 . Looking at the measures listed in the table, it is evident that the proposed controller outperforms the standard $\mathscr{L}_{1}$ adaptive control and shows no overshoot.

Table 1. Comparing the performance of the proposed and traditional adaptive controllers for a single link robot.

\begin{tabular}{ccc}
\hline Characteristics & Extended $\mathscr{L}_{1}$ adaptive back-stepping controller with BLF & $\mathscr{L}_{1}$ adaptive controller ${ }^{25}$ \\
\hline \hline Settling time $(\mathrm{sec})$ & 7.16 & 7.25 \\
\hline Overshoot $(\%)$ & $0 \%$ & $5.4 \%$ \\
\hline TR & 13.8210 & 20.1791 \\
\hline IAE & 33.1342 & 41.3859 \\
\hline RMSE & 1.1367 & 1.4313 \\
\hline
\end{tabular}


Extended $\mathcal{L}_{1}$ Adaptive Back-Stepping Controller with BLF

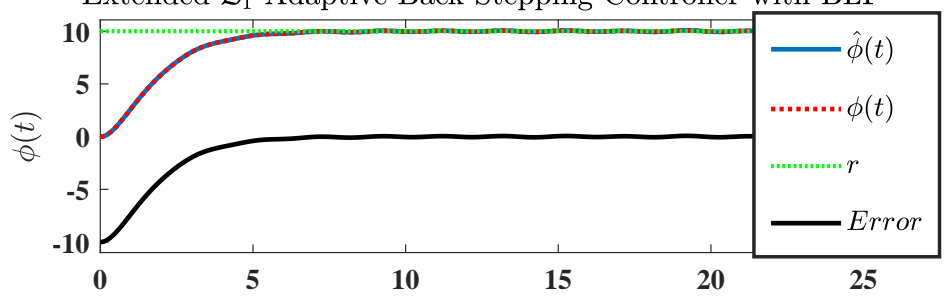

$\mathcal{L}_{1}$ Adaptive Controller ${ }^{25}$

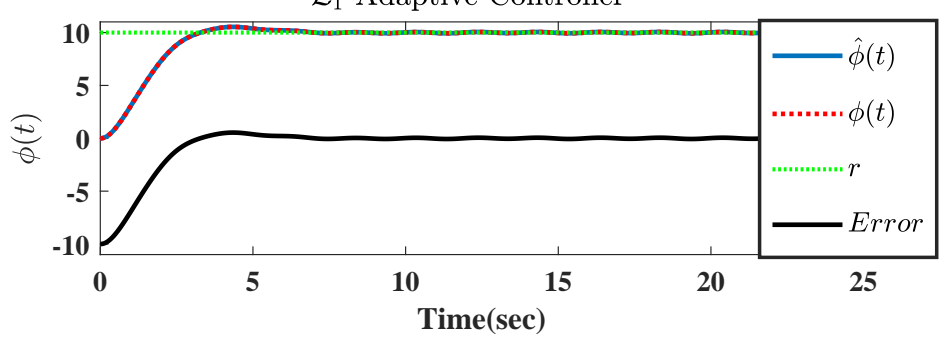

Figure 3. Performance of the proposed method (top) and the traditional method (bottom) for a single-link robot.

\section{Case study II}

In the second scenario, we consider the dynamic model of a 6DOF submersible ROV as mentioned in (3)

$$
\begin{aligned}
& \dot{\mathbf{x}}_{\mathbf{1}}=\mathbf{J}\left(\mathbf{x}_{\mathbf{1}}\right) \mathbf{x}_{\mathbf{2}} \\
& \dot{\mathbf{x}}_{\mathbf{2}}=\underbrace{-\mathbf{M}^{-\mathbf{1}} \mathbf{D}_{\mathbf{L}} \mathbf{x}_{\mathbf{2}}}_{\mathbf{A}^{\prime}}+\underbrace{\mathbf{M}^{-\mathbf{1}}}_{\mathbf{b}}(\mathbf{u}+\underbrace{\left(-\mathbf{C}\left(\mathbf{x}_{\mathbf{2}}\right)-\mathbf{D}_{\mathbf{n L}}\left(\mathbf{x}_{\mathbf{2}}\right)\right) \mathbf{x}_{\mathbf{2}}-\mathbf{g}\left(\mathbf{x}_{\mathbf{1}}\right)}_{\mathbf{f}_{\mathbf{1}}\left(\mathbf{x}_{\mathbf{1}}(t), \mathbf{x}_{\mathbf{2}}(t), t\right)}) \quad \mathbf{y}(t)=\mathbf{C x}(t)
\end{aligned}
$$

The parameters used to simulate the dynamic system above are listed in Table 2. It is worth pointing out that accurate tracking of the desired trajectory requires an additional element, the so called gain tuning. In the single-input single-output systems, a feed-forward gain $k$ is required to eliminate the effect of the steady-state error. Nonetheless, in the multivariable systems this gain does not necessarily lead to a minimum steady-state tracking error due to the interaction between different inputs/outputs. Therefore, the tuning gain is designed as

$$
\begin{aligned}
& \mathbf{K}_{g}=-\mathbf{k}_{c i}\left(\mathbf{c}\left(\mathbf{A}_{m}^{\prime}\right)^{-1} \mathbf{b}\right)^{-1} ; \mathbf{k}_{c i}=\operatorname{diag}\left[k_{c 1}, k_{c 2}, \ldots, k_{c 6}\right] \\
& 0.5 \leq k_{c i} \leq 1
\end{aligned}
$$

The values tuned for the $k_{c i}$ parameters in (57) are $k_{c 1}=1, k_{c 2}=0.95, k_{c 3}=0.95, k_{c 4}=1, k_{c 5}=0.85$, and $k_{c 6}=0.9$.

Figure 4 , illustrates the value of position error coefficient as a function of position and velocity error norms. In order to evaluate the performance of the proposed method in front of the time-varying disturbance, the disturbance profile shown in Figure 5 is applied to the system. The performance of the system to follow a fixed velocity and position setpoint along with the estimated states (without and with disturbance) are depicted in Figures 6 to 11. The results confirm a proper tracking and state estimation performance for both position and velocity variables of the ROV in presence of the time-varying disturbances and nonlinear uncertainties. To gain a better assessment on the quality of the controller, the control efforts are also plotted in Figures 8 and 11. These figures demonstrate the control signals designed for the proposed controller (without and with disturbance) to compensate and direct the ROV to follow the desired velocity and position values. As can be seen from these figures, the control signals in both cases represent a smooth and well-behaved performance without any drastic changes. It is worth noting that the control signal in (40), can be divided into two components. The second component is dealing with compensation of the steady-state error and represents the feedforward gain of the control signal. Since the reference signal $\mathbf{r}(t)$ is assumed to be constant in the simulation results, the feedforward matrix $\mathbf{K}_{g}$ is also a constant matrix, and hence the second part of the control signal is always constant. The value of the first component of the control signal is calculated according to the term $-\hat{\theta}^{T}\|\mathbf{x}(t)\|_{\mathscr{L}_{\infty}}-\hat{\sigma}(t)+\hat{\mu}(t) \mathbf{A}_{1}^{T} \xi(t)+\mathbf{e}_{\mathbf{x}} \frac{1+\tan ^{2}\left(K \mathbf{e}_{\mathbf{x}}{ }^{T} \mathbf{e}_{\mathbf{x}}\right)}{1+\tan ^{2}\left(K \mathbf{e}_{\mathbf{v}}{ }^{2} \mathbf{e}_{\mathbf{v}}\right)}+k_{v} \mathbf{e}_{\mathbf{v}}$. Since the error signal converges to zero gradually, this term is shrinking and its value will become negligible compared to the second term. This justifies clearly, why the control signal shows almost a constant value during the adaptation. 


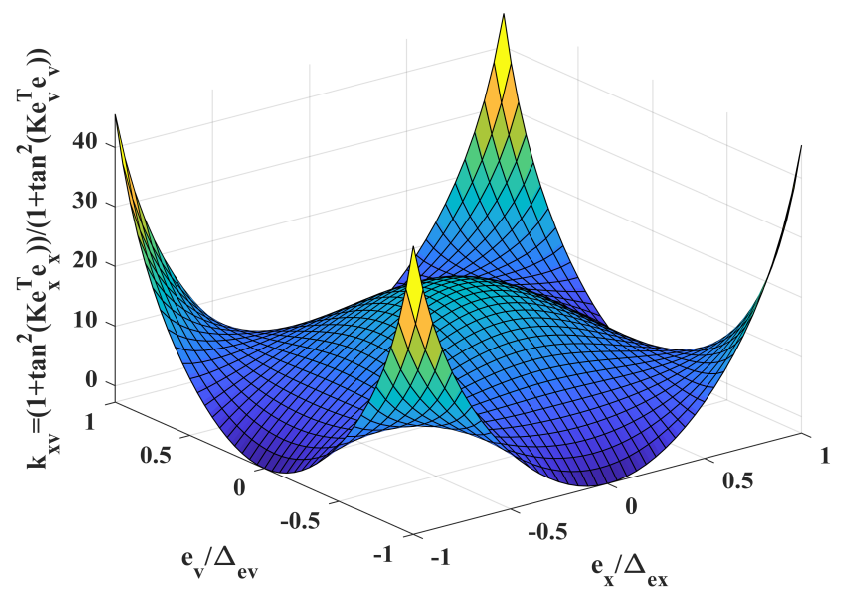

Figure 4. The position error coefficient in the control signal.

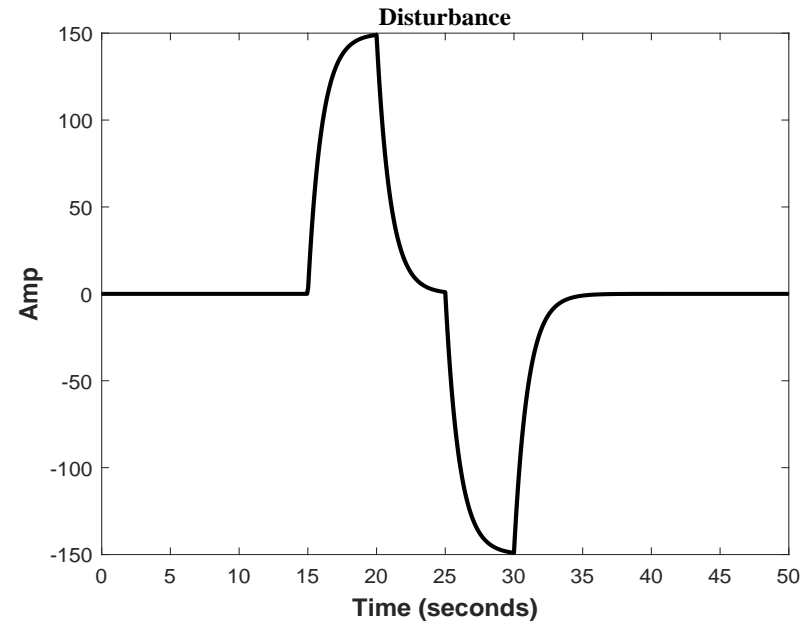

Figure 5. The time-varying disturbance profile applied to the system.

Table 2. The parameters used for the numerical simulation in case study II.

\begin{tabular}{cc||cc}
\hline $\mathrm{m}$ & $405 \mathrm{~kg}$ & $\Gamma$ & 500000 \\
\hline $\mathbf{e}_{\mathbf{x}}(\mathbf{0})$ & {$[0.5 ; 0.5 ; 0.4 ; 0 ; 0.36 ; 0.4]^{T}$} & $\mathbf{e}_{\mathbf{v}}(\mathbf{0})$ & {$[0.25 ; 0.25 ; 0.2 ; 0 ; 0.18 ; 0.2]^{T}$} \\
\hline $\mathbf{A}_{m}^{\prime}$ & $-2 \mathbf{I}_{\mathbf{6}}$ & $\varpi$ & 1.1 \\
\hline$\Delta_{e_{x}}$ & 1.1 & $\Delta_{e_{v}}$ & 0.9 \\
\hline$k_{x}$ & 0.5 & $\mathbf{k}_{v}$ & $850 \mathbf{I}_{\mathbf{6}}$ \\
\hline $\mathbf{r}_{p}(t)$ & {$[1 ; 1 ; 0 ; 0 ; 0 ; 0]^{T}$} & $\mathbf{r}_{v}(t)$ & {$[1 ; 1 ; 0.5 ; 0.2 ; 0.1 ; 1]^{T}$} \\
\hline
\end{tabular}

The tracking performance of the proposed controller is assessed by forcing the ROV to follow the step changes in the desired position in $\mathrm{x}$ and $\mathrm{y}$ directions. As can be seen from Figures 12 and 13, the step changes with amplitudes of 2, 5, 7, -2 and 0 and $-1,4,6,2$ and 0 at the time periods of 0-20, 20-40, 40-60, 60-80, and 80-100 seconds are applied to $\mathrm{x}$ and $\mathrm{y}$ directions of the ROV. The desired setpoint for other states of the ROV is assumed to be zero. The tracking performance of the proposed controller in $\mathrm{x}$ and $\mathrm{y}$ directions is shown in Figure 12. As can be inferred from this figure, the tracking is carried out with the least possible error despite the frequent changes on the desired setpoint at different time intervals. The desired trajectory tracking performance of the submarine on the x-y plane is depicted in Figure 13.

For further evaluation the performance of the proposed controller is compared against the standard $\mathscr{L}_{1}$ adaptive control method in Figures 14 and 15. The standard $\mathscr{L}_{1}$ adaptive controller is designed after fine tuning of the low-pass filters parameters as below

$$
\begin{aligned}
& C_{1}(s)=\frac{k_{1}}{s+k_{1}}=\frac{1}{\frac{1}{k_{1}} s+1}=\frac{1}{\tau_{1} s+1} ; C_{2}(s)=\frac{1}{\tau_{2} s+1} \\
& C_{3}(s)=\frac{1}{\tau_{3} s+1} ; C_{4}(s)=\frac{1}{\tau_{4} s+1} ; C_{5}(s)=\frac{1}{\tau_{5} s+1} \\
& C_{6}(s)=\frac{1}{\tau_{6} s+1} ;\left\{\tau_{1}=0.0025 ; \tau_{2}=0.005 ; \tau_{3}=0.005 ; \tau_{4}=0.0025 ; \tau_{5}=0.0014 ; \tau_{6}=0.002\right\} .
\end{aligned}
$$

However, the other simulation parameters remain unchanged according to Table 2. Comparing these figures with the results shown in Figures 7 and 8, shows evidently that the proposed method has a better performance than the standard $\mathscr{L}_{1}$ adaptive control. The result is justified further through the numerical analysis of the velocity error listed in Table 3 . As can be seen from the table, the proposed method outperforms the standard $\mathscr{L}_{1}$ adaptive control for all different linear and angular velocities. Moreover, comparing the control efforts of the standard $\mathscr{L}_{1}$ adaptive control with the control efforts of the proposed method in Figures 16 and 8 confirms that the proposed method consumes lower energy overall. 

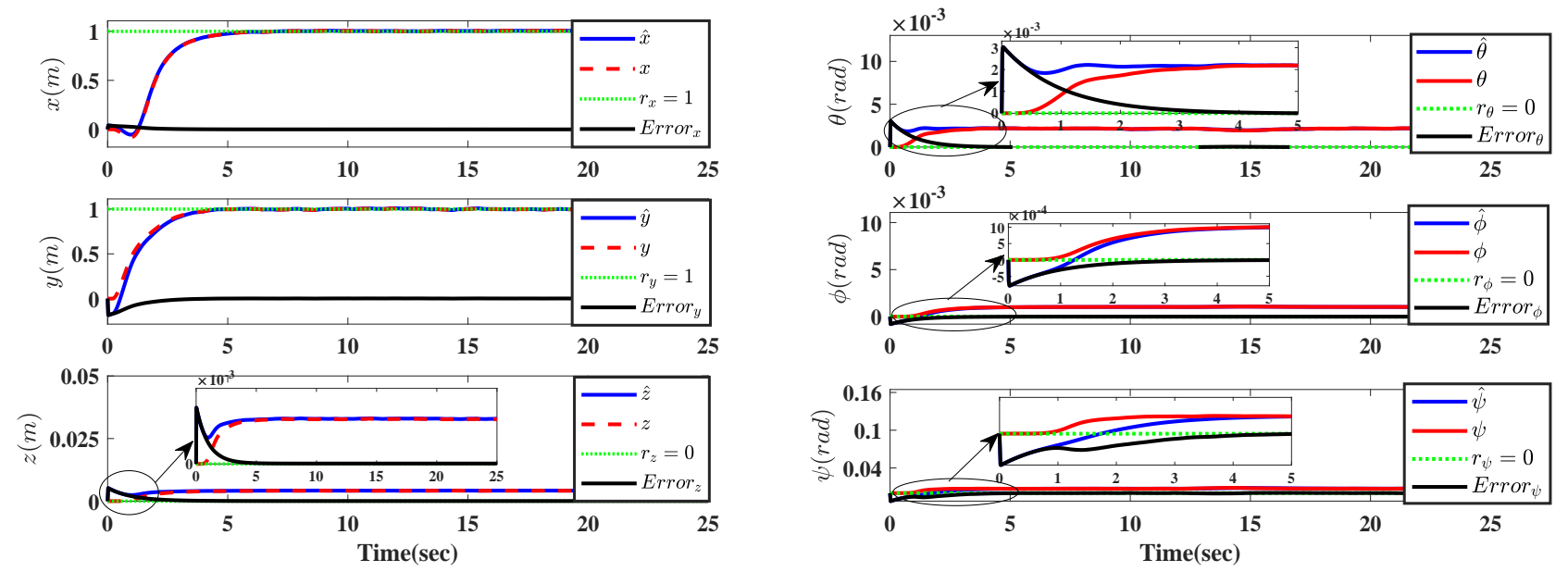

Figure 6. The closed-loop system response to follow a fixed position reference input along with the estimated states for the proposed $\mathscr{L}_{1}$ adaptive back-stepping with BLF controller (without disturbance in the system input).
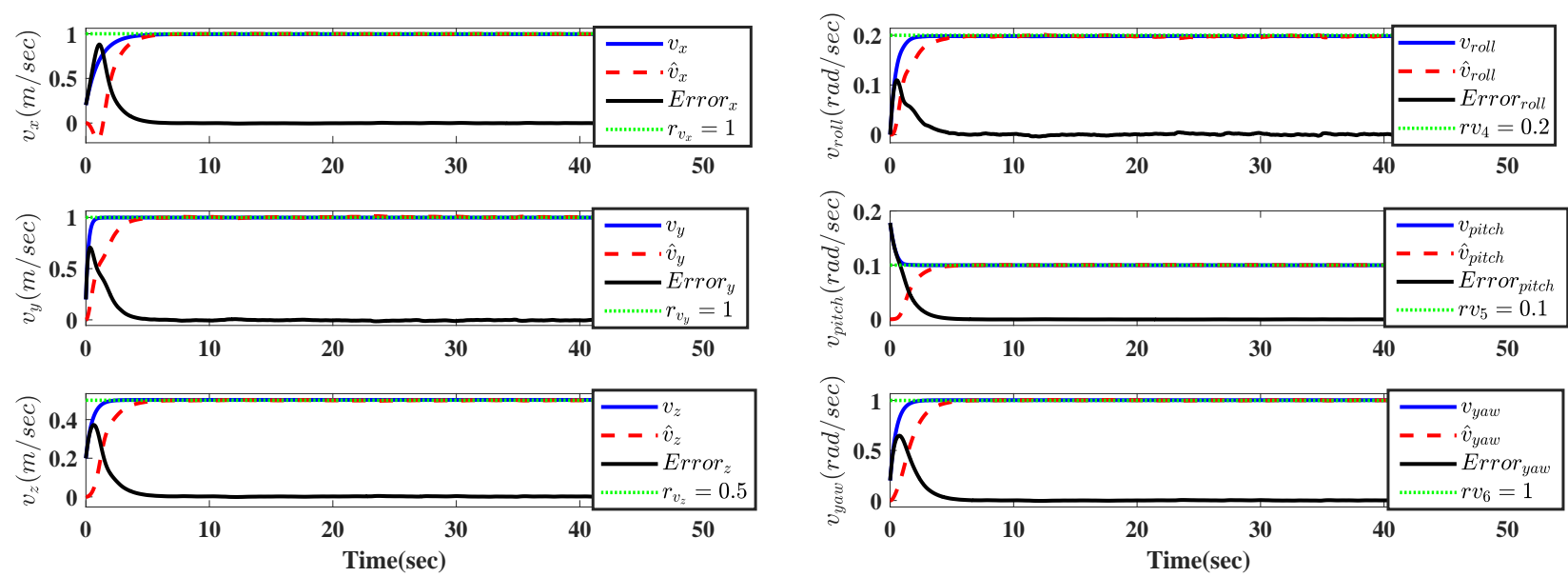

Figure 7. The closed-loop system response to follow a fixed velocity reference input along with the estimated states for the proposed $\mathscr{L}_{1}$ adaptive back-stepping with BLF controller (without disturbance in the system input).

Table 3. Comparing the performance of the proposed and standard $\mathscr{L}_{1}$ adaptive control for the ROV robot.

\begin{tabular}{|c|c|c|c|c||c|c|c|c|}
\hline \multirow{2}{*}{ Method } & \multicolumn{4}{|c||}{$\mathscr{L}_{1}$ adaptive controller } & \multicolumn{4}{c|}{$\mathscr{L}_{1}$ Adaptive Back-Stepping with BLF Controller } \\
\cline { 2 - 8 } & TR & IAE & Overshoot(\%) & Settling time(sec) & TR & IAE & Overshoot(\%) & Settling time(sec) \\
\hline \hline$e_{v_{x}}$ & 3.4054 & 6.8107 & $52.1 \%$ & 5.967 & 1.5374 & 3.4053 & $0 \%$ & 5.151 \\
\hline$e_{v_{y}}$ & 2.860 & 5.2350 & $0 \%$ & 5.967 & 1.0229 & 2.6869 & $0 \%$ & 4.063 \\
\hline$e_{v_{z}}$ & 0.6542 & 1.3648 & $0 \%$ & 5.219 & 0.7489 & 1.5074 & $0 \%$ & 5.049 \\
\hline$e_{v_{\text {roll }}}$ & 0.5510 & 1.2844 & $3.23 \%$ & 5.219 & 0.1885 & 0.4872 & $0 \%$ & 4.675 \\
\hline$e_{v_{\text {pitch }}}$ & 0.2621 & 0.5377 & $0 \%$ & 5.151 & 0.2062 & 0.4355 & $0 \%$ & 4.335 \\
\hline$e_{v_{\text {yaw }}}$ & 1.3839 & 3.8654 & $0 \%$ & 5.83 & 1.3527 & 2.7878 & $0 \%$ & 4.947 \\
\hline$e_{v_{\text {total }}}$ & 9.1166 & 19.098 & $55.33 \%$ & 33.353 & 5.0566 & 11.3101 & $0 \%$ & 28.22 \\
\hline
\end{tabular}

\section{Discussion and Conclusions}

In this paper, an $\mathscr{L}_{1}$ adaptive back-stepping control with BLF and predefined constraints for the position-velocity is proposed. The purpose of this investigation is to remove the effect of the continuous linear filter in the whole design process, and hence 

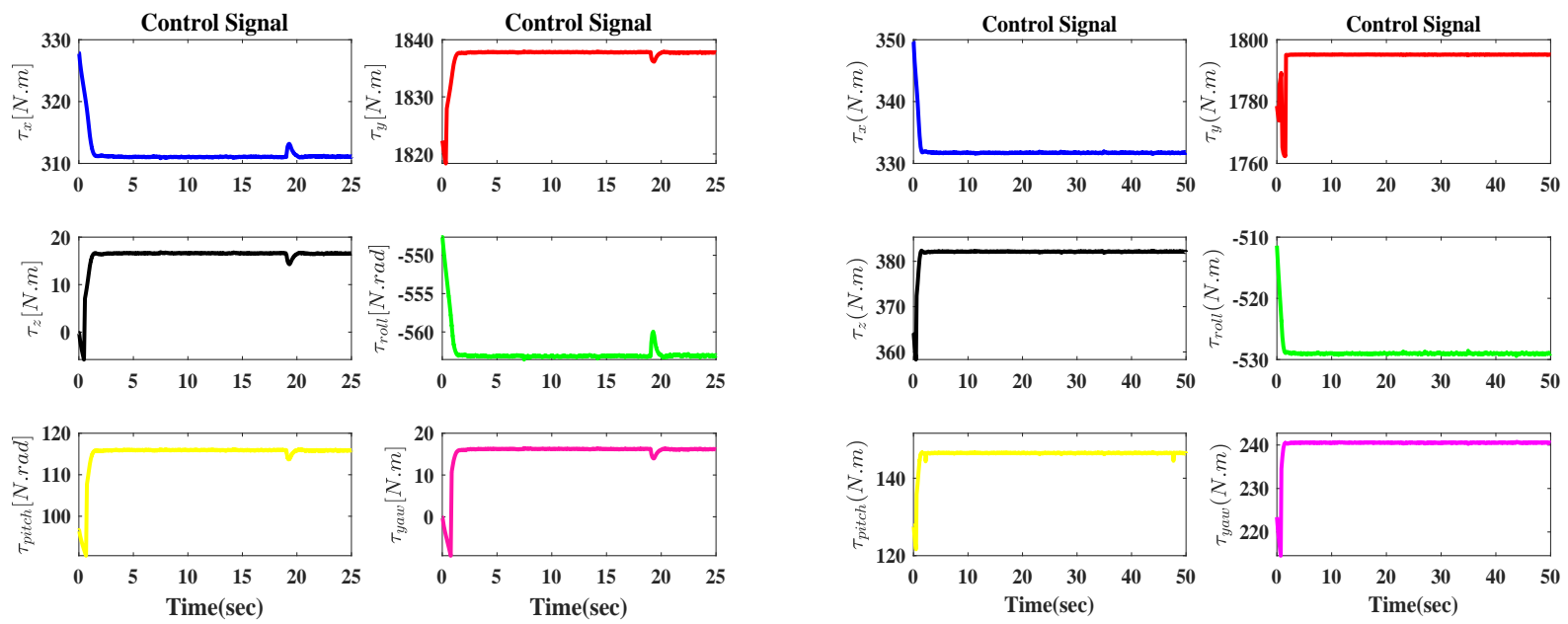

(a)

(b)

Figure 8. The $\mathscr{L}_{1}$ adaptive back-stepping with BLF control signals for ROV robot (without disturbance in the system input): (a) Position control signal, (b) Velocity control signal.
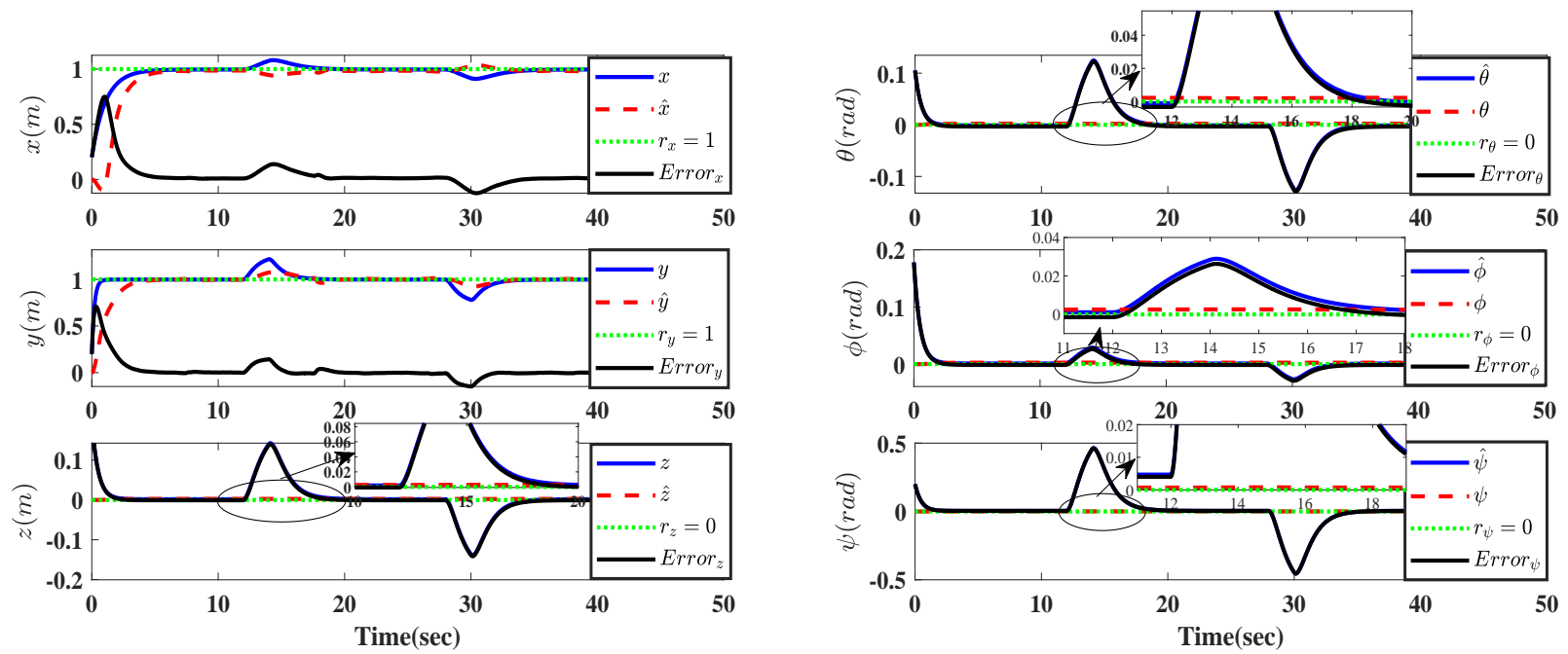

Figure 9. The closed-loop system response to follow a fixed position reference input along with the estimated states for the proposed $\mathscr{L}_{1}$ adaptive back-stepping with BLF controller (with disturbance in the system input).

reduce the complexity of the design in the standard $\mathscr{L}_{1}$ adaptive control. In this way, a fast adaptation and robustness is guaranteed. In the proposed technique, a pre-defined set of constraints on the velocity and position of the system is imposed by the use of barrier Lyapunov functions through which a new adaptation law is designed. In this way, it is possible to guarantee that the tracking errors of the proposed method stay inside the known constraints despite the unknown and timevarying parameters and nonlinear uncertainties acting on the system. The efficacy of the proposed technique is evaluated on a 6-DOF underwater ROV robot as well as a single-link robotic manipulator. The achieved results demonstrate a very good tracking and state estimation performance for both position and velocity states of the system. The results are evaluated despite the presence of time-varying disturbances and uncertainties on a nonlinear dynamic of the system. As the future work, the problem of lack of access to system states and designing an observer is going to be investigated. 

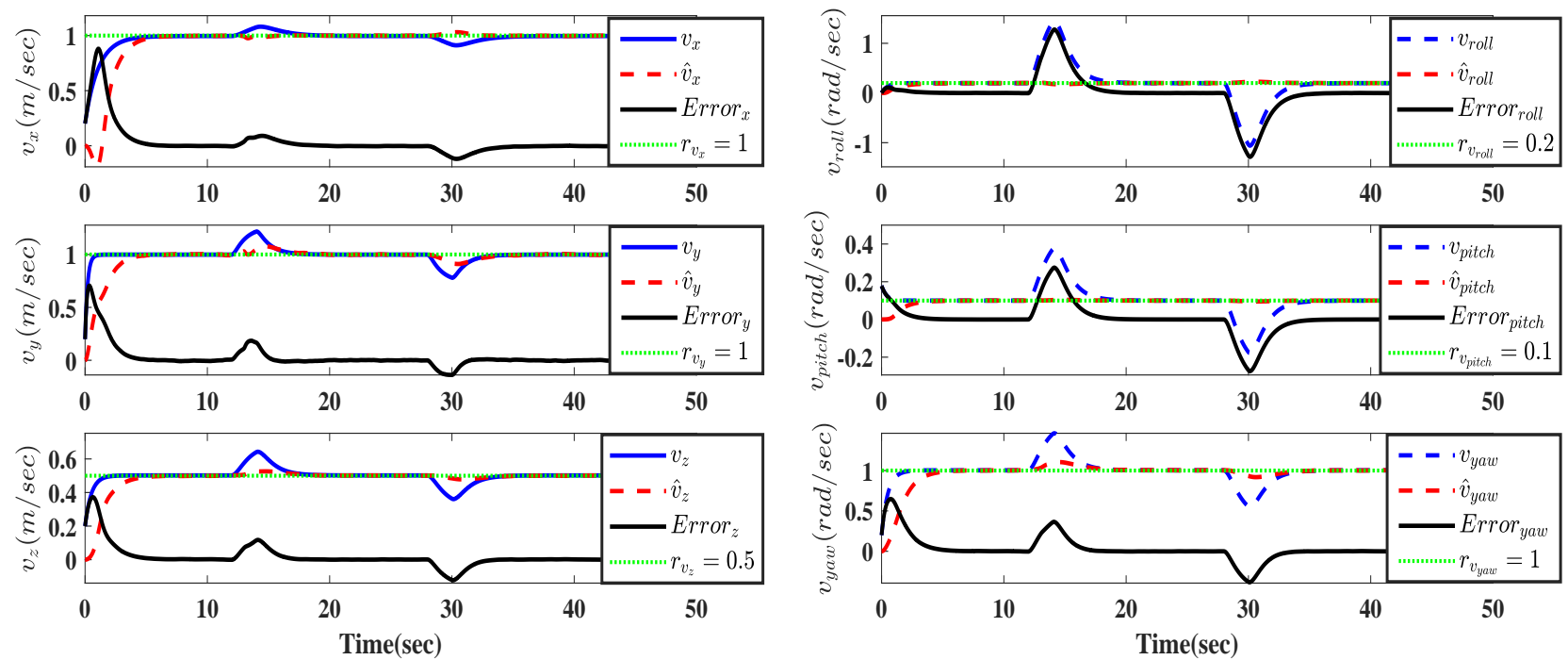

Figure 10. The closed-loop system response to follow a fixed velocity reference input along with the estimated states for the proposed $\mathscr{L}_{1}$ adaptive back-stepping with BLF controller (with disturbance in the system input).
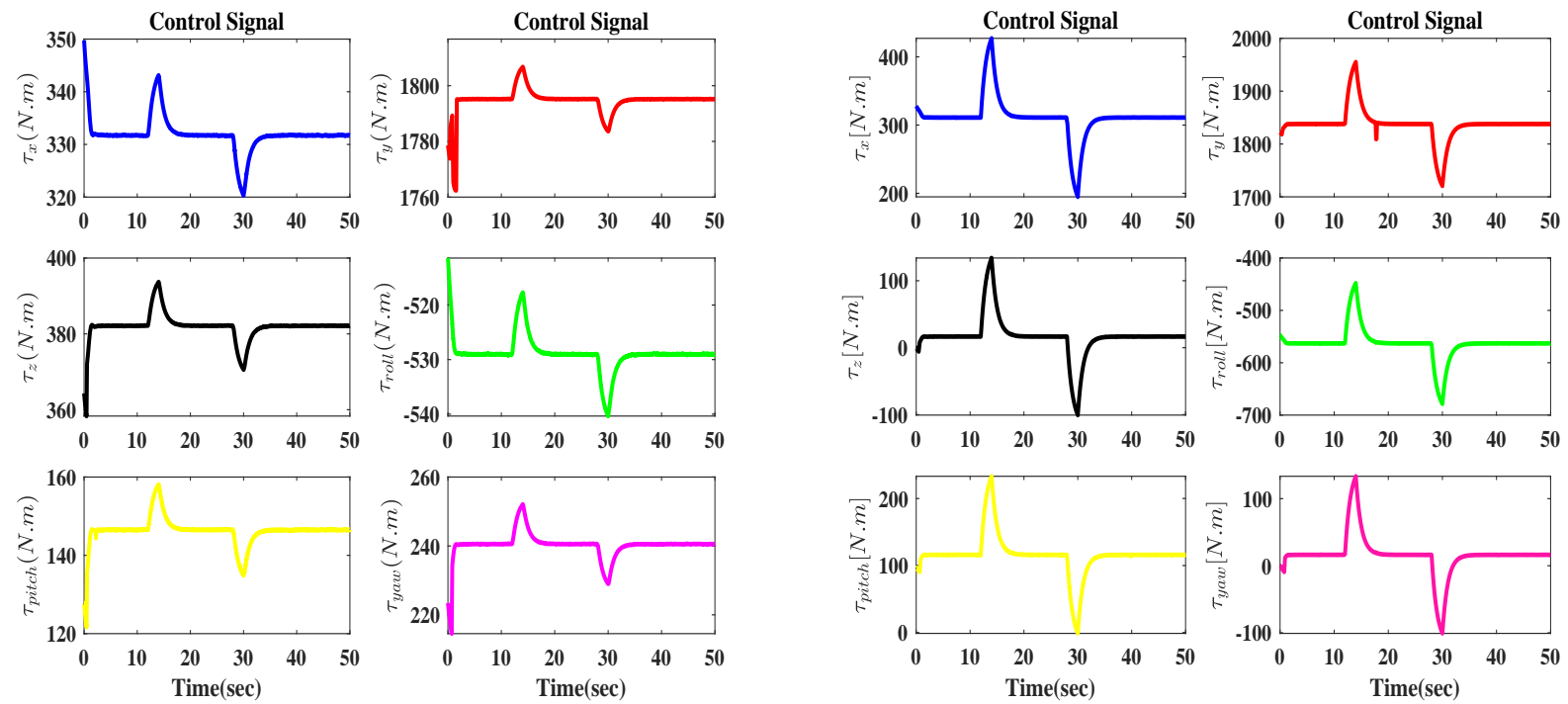

(a)

(b)

Figure 11. The $\mathscr{L}_{1}$ adaptive back-stepping with BLF control signals for ROV robot (with disturbance in the system input) (a) position control effort, (b) velocity control effort. 


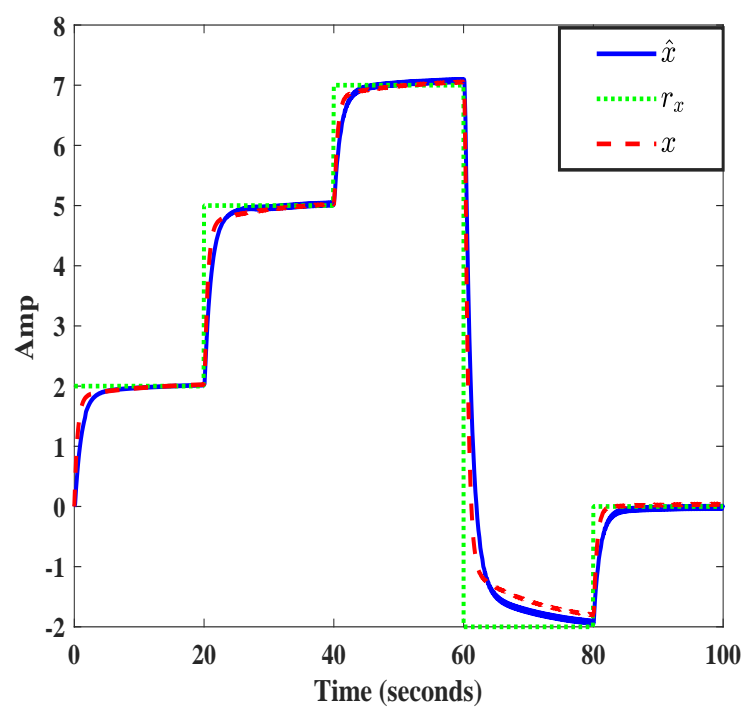

(a)

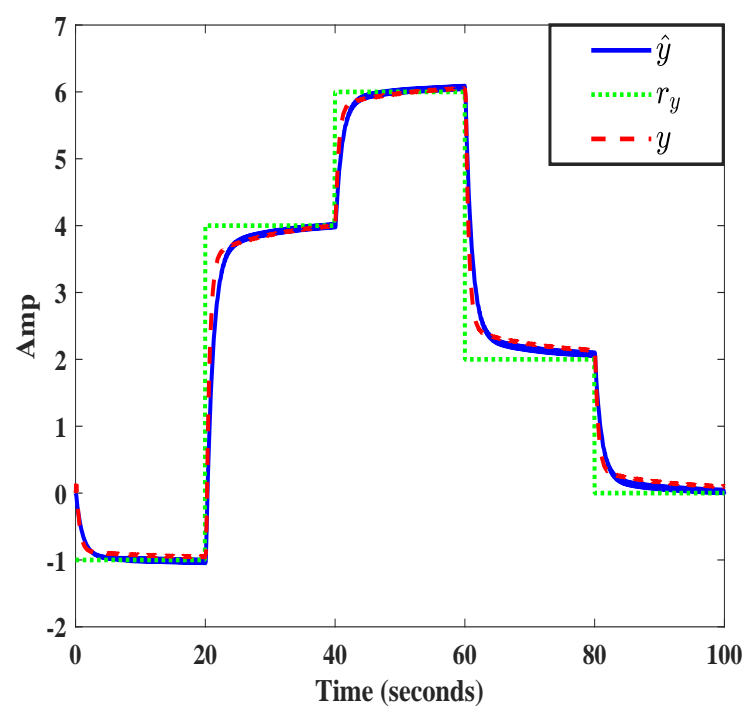

(b)

Figure 12. The tracking performance of the ROV positions for frequent step changes in $x(a)$ and $y$ (b) directions.

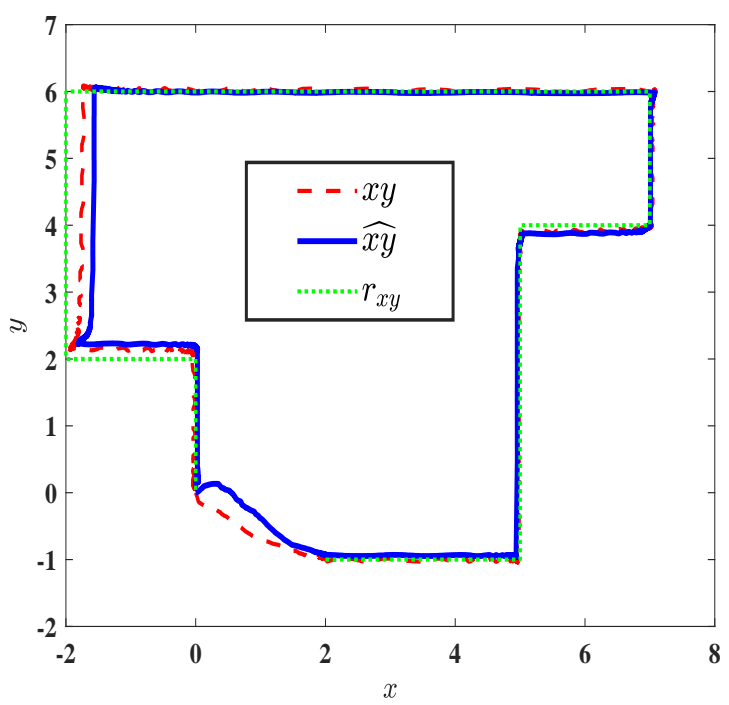

Figure 13. The tracking performance of the desired trajectory the submarine on the $x-y$ plane. 

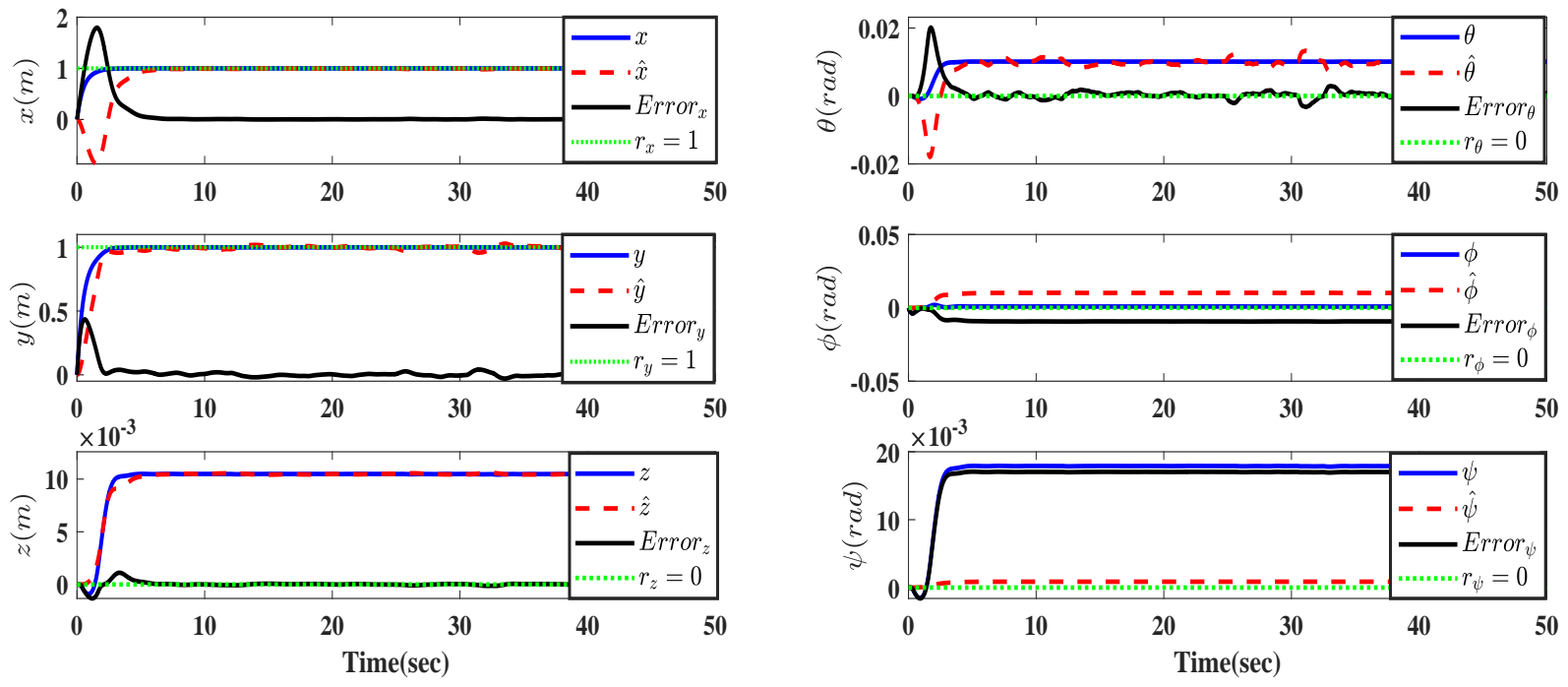

Figure 14. The closed-loop system response to follow a fixed position reference input along with the estimated states for the $\mathscr{L}_{1}$ adaptive controller.
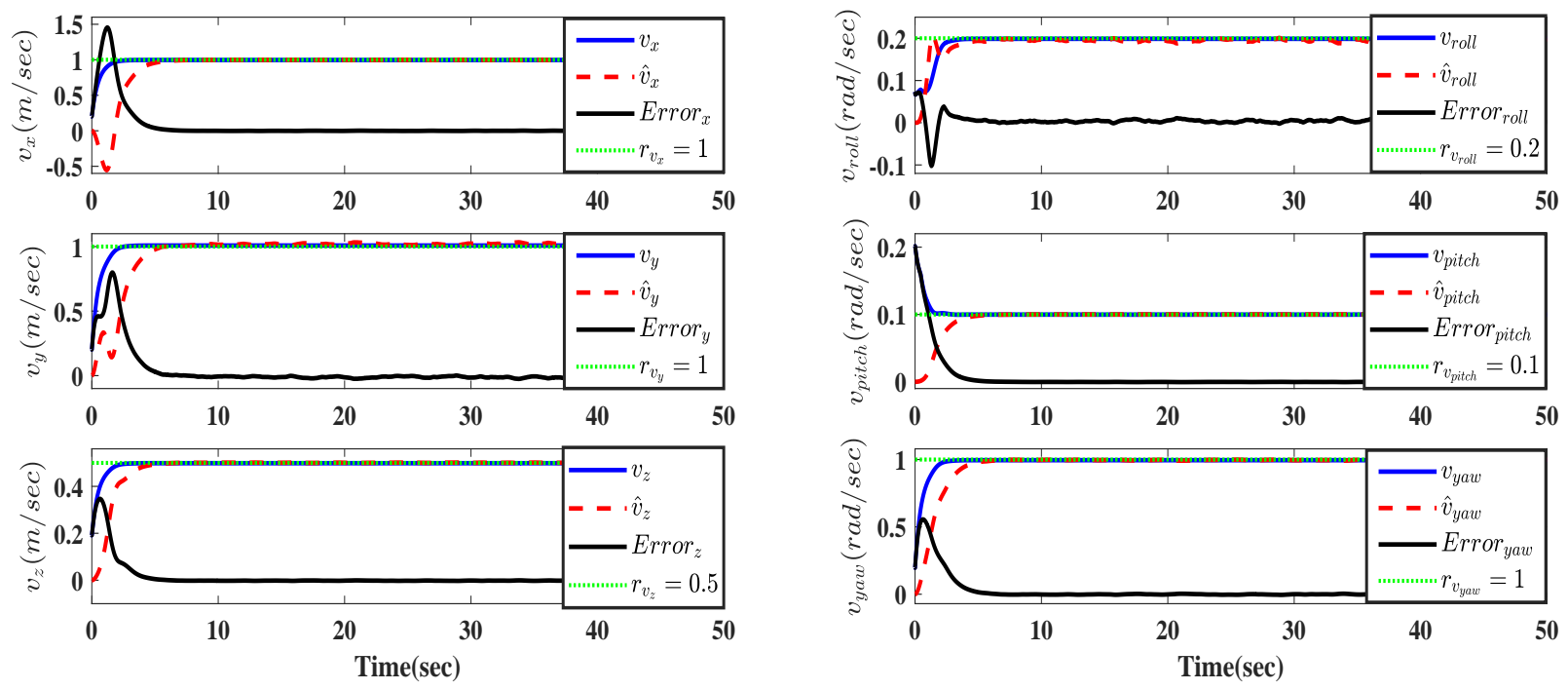

Figure 15. The closed-loop system response to follow a fixed velocity reference input along with the estimated states for the $\mathscr{L}_{1}$ adaptive controller. 

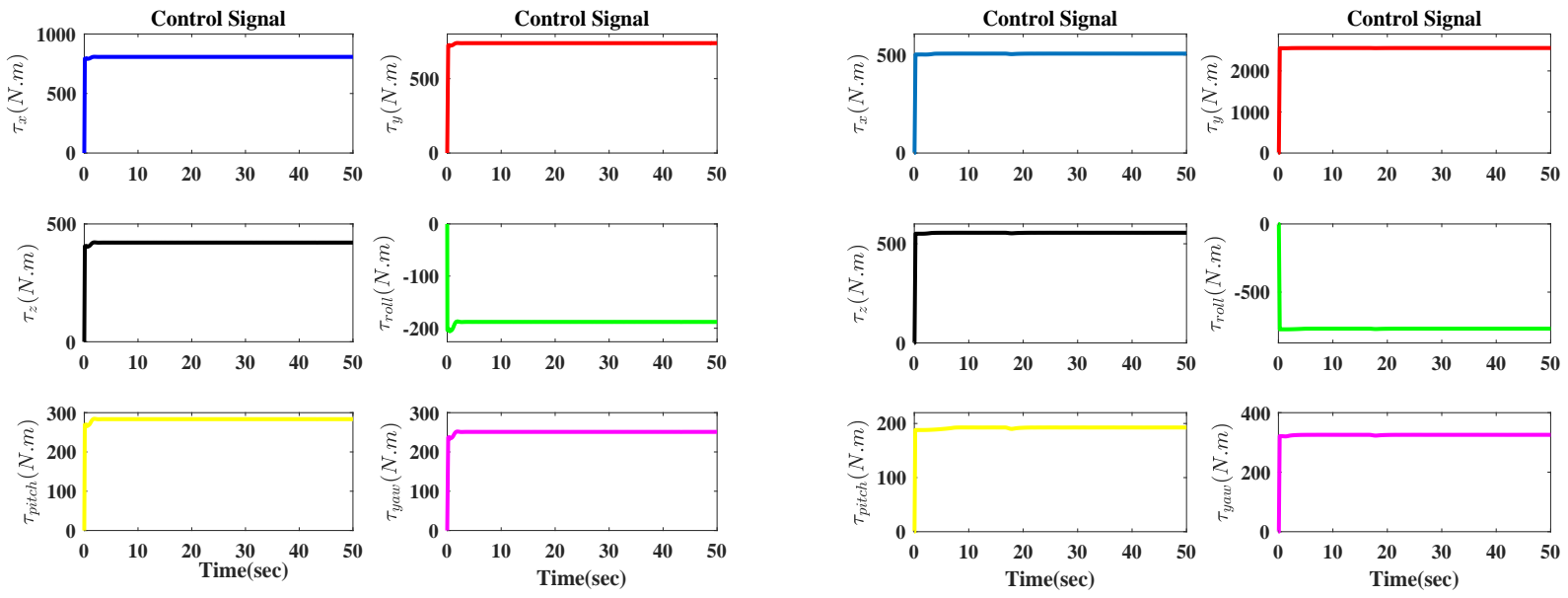

(a)

(b)

Figure 16. The control effort of the standard $\mathscr{L}_{1}$ adaptive control for the ROV robot: (a) Position control signal, (b) Velocity control signal .

\section{References}

1. Burrell, T., Montazeri, A., Monk, S. \& Taylor, C. J. Feedback controlbased inverse kinematics solvers for a nuclear decommissioning robot. IFAC-PapersOnLine 49, 177-184, DOI: https://doi.org/10.1016/j.ifacol.2016.10.541 (2016).

2. Montazeri, A. \& Ekotuyo, J. Development of dynamic model of a 7 dof hydraulically actuated tele-operated robot for decommissioning applications. In 2016 American Control Conference (ACC), 1209-1214, DOI: https://doi.org/10.1109/ ACC.2016.7525082 (2016).

3. Nemati, H. \& Montazeri, A. Design and development of a novel controller for robust attitude stabilisation of an unmanned air vehicle for nuclear environments. In 2018 UKACC 12th International Conference on Control (CONTROL), 373-378, DOI: https://doi.org/10.1109/CONTROL.2018.8516729 (2018).

4. Makavita, C. D., Jayasinghe, S. G., Nguyen, H. D. \& Ranmuthugala, D. Experimental study of a command governor adaptive depth controller for an unmanned underwater vehicle. Appl. Ocean. Res. 86, 61-72, DOI: https://doi.org/10. 1016/j.apor.2019.02.016 (2019).

5. Gao, J., Wu, P., Li, T. \& Proctor, A. Optimization-based model reference adaptive control for dynamic positioning of a fully actuated underwater vehicle. Nonlinear Dyn. 87, 2611-2623, DOI: https://doi.org/10.1007/s11071-016-3214-2 (2017).

6. Dong, M., Li, J. \& Chou, W. Depth control of rov in nuclear power plant based on fuzzy pid and dynamics compensation. Microsyst. Technol. 26, 811-821, DOI: https://doi.org/10.1007/s00542-019-04605-x (2020).

7. Phillips, B. T. et al. A dexterous, glove-based teleoperable low-power soft robotic arm for delicate deep-sea biological exploration. Sci. reports 8, 1-9, DOI: https://doi.org/10.1038/s41598-018-33138-y (2018).

8. Fischer, N., Bhasin, S. \& Dixon, W. Nonlinear control of an autonomous underwater vehicle: A rise-based approach. In Proceedings of the 2011 American Control Conference, 3972-3977, DOI: https://doi.org/10.1109/ACC.2011.5990958 (2011).

9. Schjølberg, I. \& Fossen, T. I. Modelling and control of underwater vehicle-manipulator systems. In in Proc. rd Conf. on Marine Craft maneuvering and control (1994).

10. Antonelli, G., Chiaverini, S. \& Sarkar, N. External force control for underwater vehicle-manipulator systems. IEEE Transactions on Robotics Autom. 17, 931-938, DOI: https://doi.org/10.1109/70.976027 (2001).

11. Maalouf, D., Creuze, V. \& Chemori, A. A novel application of multivariable $\mathscr{L}_{1}$ adaptive control: From design to realtime implementation on an underwater vehicle. In 2012 IEEE/RSJ international conference on intelligent robots and systems, 76-81, DOI: https://doi.org/10.1109/IROS.2012.6385498 (2012). 
12. Maalouf, D., Chemori, A. \& Creuze, V. $\mathscr{L}_{1}$ adaptive depth and pitch control of an underwater vehicle with real-time experiments. Ocean. Eng. 98, 66-77, DOI: https://doi.org/10.1016/j.oceaneng.2015.02.002 (2015).

13. Liu, S., Liu, Y. \& Wang, N. Robust adaptive self-organizing neuro-fuzzy tracking control of uuv with system uncertainties and unknown dead-zone nonlinearity. Nonlinear Dyn. 89, 1397-1414, DOI: https://doi.org/10.1007/s11071-017-3524-z (2017).

14. Shen, C., Shi, Y. \& Buckham, B. Trajectory tracking control of an autonomous underwater vehicle using lyapunov-based model predictive control. IEEE Transactions on Ind. Electron. 65, 5796-5805, DOI: https://doi.org/10.1109/TIE.2017. 2779442 (2017).

15. Nag, A., Patel, S. S., Kishore, K. \& Akbar, S. A robust h-infinity based depth control of an autonomous underwater vehicle. In 2013 International Conference on Advanced Electronic Systems (ICAES), 68-73, DOI: https://doi.org/10. 1109/ICAES.2013.6659363 (2013).

16. Liang, X., Wan, L., Blake, J. I., Shenoi, R. A. \& Townsend, N. Path following of an underactuated auv based on fuzzy backstepping sliding mode control. Int. J. Adv. Robotic Syst. 13, 122, DOI: https://doi.org/10.5772/64065 (2016).

17. Xargay, E., Hovakimyan, N. \& Cao, C. Benchmark problems of adaptive control revisited by $\mathscr{L}_{1}$ adaptive control. In 2009 17th Mediterranean Conference on Control and Automation, 31-36, DOI: https://doi.org/10.1109/MED.2009.5164510 (2009).

18. Ioannou, P. \& Fidan, B. Adaptive control tutorial (SIAM, 2006).

19. Lochan, K., Singh, J., Roy, B. \& Subudhi, B. Adaptive time-varying super-twisting global smc for projective synchronisation of flexible manipulator. Nonlinear Dyn. 93, 2071-2088, DOI: https://doi.org/10.1007/s11071-018-4308-9 (2018).

20. Ma, J., Park, J. H. \& Xu, S. Global adaptive finite-time control for uncertain nonlinear systems with actuator faults and unknown control directions. Nonlinear Dyn. 97, 2533-2545, DOI: https://doi.org/10.1007/s11071-019-05146-8 (2019).

21. Peter, F., Leitão, M. \& Holzapfel, F. Adaptive augmentation of a new baseline control architecture for tail-controlled missiles using a nonlinear reference model. In AIAA Guidance, Navigation, and Control Conference, 5037, DOI: https: //doi.org/10.2514/6.2012-5037 (2012).

22. Ahmadian, H., Talebi, H. A. \& Sharifi, I. $\mathscr{L}_{1}$ adaptive controller design for single-link flexible joint manipulator with fuzzy-pid filter. In 2020 28th Iranian Conference on Electrical Engineering (ICEE), 1-6, DOI: 10.1109/ICEE50131.2020. 9261009 (2020).

23. Cao, C. \& Hovakimyan, N. Design and analysis of a novel 11 adaptive controller, part i: control signal and asymptotic stability. In 2006 American Control Conference, 3397-3402, DOI: https://doi.org/10.1109/ACC.2006.1657243 (2006).

24. Kharisov, E., Gregory, I., Cao, C. \& Hovakimyan, N. $\mathscr{L}_{1}$ adaptive control for flexible space launch vehicle and proposed plan for flight validation. In AIAA Guidance, Navigation and Control Conference and Exhibit, 7128, DOI: https://doi.org/ 10.2514/6.2008-7128 (2008).

25. Hovakimyan, N. \& Cao, C. $\mathscr{L}_{1}$ adaptive control theory: Guaranteed robustness with fast adaptation (SIAM, 2010).

26. Kharisov, E. \& Hovakimyan, N. $\mathscr{L}_{1}$ adaptive output feedback controller for minimum phase systems. In Proceedings of the 2011 American Control Conference, 1182-1187, DOI: https://doi.org/10.1109/ACC.2011.5991012 (2011).

27. Kim, K.-K. K. \& Hovakimyan, N. Multi-criteria optimization for filter design of $\mathscr{L}_{1}$ adaptive control. J. Optim. Theory Appl. 161, 557-581, DOI: https://doi.org/10.1007/s10957-013-0412-7 (2014).

28. Antonelli, G., Caccavale, F., Chiaverini, S. \& Villani, L. Tracking control for underwater vehicle-manipulator systems with velocity estimation. IEEE J. Ocean. Eng. 25, 399-413, DOI: https://doi.org/10.1109/48.855403 (2000).

29. Ferreira, B., Pinto, M., Matos, A. \& Cruz, N. Control of the mares autonomous underwater vehicle. In OCEANS 2009, 1-10, DOI: https://doi.org/10.23919/OCEANS.2009.5422133 (2009).

30. Das, S. et al. Control architecture for auv-150: A systems approach. In FIRA RoboWorld Congress, 41-48, DOI: https://doi.org/10.1007/978-3-642-15810-0_6 (2010).

31. Zhou, S., Zhang, S. \& Wang, D. Impact angle control guidance law with seekers field-of-view constraint based on logarithm barrier lyapunov function. IEEE Access 8, 68268-68279, DOI: https://doi.org/10.1109/ACCESS.2020.2986355 (2020).

32. Lee, H., Snyder, S. \& Hovakimyan, N. $\mathscr{L}_{1}$ adaptive output feedback augmentation for missile systems. IEEE Transactions on Aerosp. Electron. Syst. 54, 680-692, DOI: https://doi.org/10.1109/TAES.2017.2764218 (2017). 
33. Zuo, Z. \& Mallikarjunan, S. $\mathscr{L}_{1}$ adaptive backstepping for robust trajectory tracking of uavs. IEEE Transactions on Ind. Electron. 64, 2944-2954, DOI: https://doi.org/10.1007/978-3-642-15810-0_6 (2016).

34. Elnaggar, M., Saad, M. S., Abdel Fattah, H. A. \& Elshafei, A. L. $\mathscr{L}_{1}$ adaptive fuzzy control of wind energy conversion systems via variable structure adaptation for all wind speed regions. IET Renew. Power Gener. 12, 18-27, DOI: https: //doi.org/10.1049/iet-rpg.2017.002 (2018).

35. Beikzadeh, H. \& Liu, G. Trajectory tracking of quadrotor flying manipulators using 11 adaptive control. J. Frankl. Inst. 355, 6239-6261, DOI: https://doi.org/10.1016/j.jfranklin.2018.06.011 (2018).

36. Maiti, R., Sharma, K. D. \& Sarkar, G. Linear consequence-based fuzzy parallel distributed compensation type $l_{-}\{1\}$ adaptive controller for two link robot manipulator. IEEE Transactions on Circuits Syst. I: Regul. Pap. 66, 3978-3990, DOI: https://doi.org/10.1109/TCSI.2019.2916255 (2019).

37. Cui, R., Chen, L., Yang, C. \& Chen, M. Extended state observer-based integral sliding mode control for an underwater robot with unknown disturbances and uncertain nonlinearities. IEEE Transactions on Ind. Electron. 64, 6785-6795, DOI: https://doi.org/10.1109/TIE.2017.2694410 (2017).

38. Li, Z., Yang, C., Ding, N., Bogdan, S. \& Ge, T. Robust adaptive motion control for underwater remotely operated vehicles with velocity constraints. Int. J. Control. Autom. Syst. 10, 421-429, DOI: https://doi.org/10.1007/s12555-012-0222-y (2012).

39. Fossen, T. I. Marine control systems-guidance. navigation, and control of ships, rigs and underwater vehicles. Mar. Cybern. Trondheim, Norway, Org. Number NO 985195005 MVA, www. marinecybernetics. com, ISBN: 8292356002 (2002).

40. Wadoo, S. \& Kachroo, P. Autonomous underwater vehicles: modeling, control design and simulation (CRC Press, 2017).

41. Tee, K. P., Ge, S. S. \& Tay, E. H. Barrier lyapunov functions for the control of output-constrained nonlinear systems. Automatica 45, 918-927, DOI: https://doi.org/10.1016/j.automatica.2008.11.017 (2009).

42. Ioannou, P. \& Fidan, B. Adaptive control tutorial (SIAM, 2006).

43. Cruz-Uribe, D. \& Neugebauer, C. Sharp error bounds for the trapezoidal rule and simpsons rule. J. Inequal. Pure Appl. Math 3, 1-22 (2002). 